JOURNAL OF

SYMPLECTIC GEOMETRY

Volume 10, Number 4, 535-561, 2012

\title{
NONCOMMUTATIVE INTEGRABILITY AND ACTION-ANGLE VARIABLES IN CONTACT GEOMETRY
}

\author{
BožIDAR JOVANOVIĆ
}

\begin{abstract}
We introduce a notion of the noncommutative integrability within a framework of contact geometry.
\end{abstract}

\section{Introduction}

In Hamiltonian mechanics solving by quadratures is closely related to the regularity of dynamics that is described in the Arnold-Liouville theorem. A Hamiltonian system on $2 n$-dimensional symplectic manifold $M$ is called integrable if it has $n$-smooth Poisson-commuting, almost everywhere independent integrals $f_{1}, f_{2}, \ldots, f_{n}$. Regular compact connected invariant manifolds of the system are Lagrangian tori. Moreover, in a neighborhood of any torus, there exist canonical action-angle coordinates $(\varphi, I)=$ $\left(\varphi_{1}, \ldots, \varphi_{n}, I_{1}, \ldots, I_{n}\right)$, integrals $f_{i}$ depend only on actions $I$ and the flow is translation in $\varphi$ coordinates $[\mathbf{1}]$.

Therefore, an integrable Hamiltonian system can be considered as a toric Lagrangian fibration $\pi: M \rightarrow W$ (see Duistermaat [11]). This approach is reformulated to contact manifolds $(M, \mathcal{H})$ by Banyaga and Molino [2]. Instead of a toric Lagrangian fibration, one consider an invariant toric fibration transversal to the contact distribution $\mathcal{H}$, such that intersection of tori and $\mathcal{H}$ is a Lagrangian distribution with respect to the conformal class of the symplectic structure on $\mathcal{H}$ (see Section 5).

Slightly different notion of a contact integrability is given recently by Khesin and Tabachnikov [19]. They defined integrability in terms of the existence of an invariant foliation $\mathcal{F}$, called a co-Legendrian foliation (here we refer to $\mathcal{F}$ as a pre-Legendrian foliation). $\mathcal{F}$ is transversal to $\mathcal{H}, \mathcal{G}=\mathcal{F} \cap \mathcal{H}$ is a Legendrian foliation of $M$ with an additional property that on every

Mathematics Subject Classification. 53D35, 37J35, 37J55.

Keywords and phrases. noncommutative integrability, $\alpha$-complete foliations, isotropic foliations, contact flows, action-angle coordinates. 
leaf $F$ of $\mathcal{F}$, the foliation $\left.\mathcal{G}\right|_{F}$ has a holonomy invariant transverse smooth measure. ${ }^{1}$ It turns out that this condition implies the existence of a global contact form $\alpha$ (see [19]) and $\mathcal{G}$ is a $\alpha$-complete Legendrian foliation studied by Libermann [27] and Pang [32]. Recall that a foliation $\mathcal{F}$ is $\alpha$-complete if for any pair $f_{1}, f_{2}$ of first integrals of $\mathcal{F}$ (where $f_{i}$ may be a constant), the Jacobi bracket $\left[f_{1}, f_{2}\right]$ is also a first integral of $\mathcal{F}$ (eventually a constant).

Due to the presence of symmetries, many Hamiltonian systems have more than $n$ noncommuting integrals. Illustrative examples are $G$-invariant geodesic flows on homogeneous spaces $[4, \mathbf{1 6}]$. An appropriate framework for the study of these systems is noncommutative integrability introduced by Nehoroshev $[\mathbf{3 1}]$ and Mishchenko and Fomenko $[\mathbf{3 0}]$ (see also $[\mathbf{4}, \mathbf{1 6}, \mathbf{2 1}, \mathbf{3 6}]$ ). Here, we recall the Nehoroshev formulation: a Hamiltonian system on $2 n$ dimensional symplectic manifold $M$ is noncommutatively integrable if it has $2 n-r$ almost everywhere independent integrals $f_{1}, f_{2}, \ldots, f_{2 n-r}$ and $f_{1}, \ldots, f_{r}$ commute with all integrals

$$
\left\{f_{i}, f_{j}\right\}=0, \quad i=1, \ldots, 2 n-r, \quad j=1, \ldots, r .
$$

Regular compact connected invariant manifolds of the system are isotropic tori. In a neighborhood of a regular torus, there exist canonical generalized action-angle coordinates such that integrals $f_{i}, i=1, \ldots, r$ depend only on actions and the flow is translation in angle coordinates.

One of the basic examples of contact manifolds are unit co-sphere bundles $S Q \subset T^{*} Q$ of Riemannian manifolds $(Q, g)$. The restriction of a geodesic flow to $S Q$ is a contact flow of the Reeb vector field of the associated contact form. It is clear that noncommutatively integrable geodesic flows, considered as Reeb vector flows, have a geometrical structure that need to be described by a noncommutative variant of integrability.

We introduce an appropriate concept of a contact noncommutative integrability.

In the first part of the paper (Sections 3 and 4 ), foliations on contact manifolds $(M, \mathcal{H})$ are considered. We refer to a foliation $\mathcal{F}$ as pre-isotropic if it is transversal to $\mathcal{H}$ and $\mathcal{G}=\mathcal{F} \cap \mathcal{H}$ is an isotropic subbundle of $\mathcal{H}$.

Let $\mathcal{F}$ be a pre-isotropic foliation containing the Reeb vector field $Z$ on a co-oriented contact manifold $(M, \alpha)$. The foliation $\mathcal{F}$ is $\alpha$-complete if and only if $\mathcal{E}$ is completely integrable, where $\mathcal{E}=\mathcal{F}^{\perp}$ is the pseudo-orthogonal distribution of $\mathcal{F}$ and we have a flag of foliations $\mathcal{G} \subset \mathcal{F} \subset \mathcal{E}$. Furthermore, each leaf of $\mathcal{G}$ and $\mathcal{F}$ has an affine structure (Theorem 3.2).

Thus, if $\mathcal{F}$ has compact leaves, they are tori. Locally, in an invariant neighborhood of any leaf, the foliation $\mathcal{F}$ can be seen as a fibration over some base manifold. Also, affine translations provide an Abelian Lie algebra of contact transformations with orbits that coincide with $\mathcal{F}$.

\footnotetext{
${ }^{1}$ Throughout the paper we use the same notation for foliations and their integrable distributions of tangent spaces.
} 
Next, we consider a pre-isotropic foliation $\mathcal{F}$ on a contact manifold $(M, \mathcal{H})$ with the mentioned properties of $\alpha$-complete pre-isotropic foliations: $\mathcal{F}$ is defined via submersion $\pi: M \rightarrow W$ and it is given an Abelian Lie algebra of contact symmetries $\mathcal{X}$ with orbits equal to $\mathcal{F}$. We refer to a triple $(M, \mathcal{H}, \mathcal{X})$ as a complete pre-isotropic contact structure.

For a given complete pre-isotropic structure $(M, \mathcal{H}, \mathcal{X})$, locally, there always exists an invariant contact form $\alpha$ such that $\mathcal{F}$ is $\alpha$-complete (Theorem 4.1). Note, if $\mathcal{F}$ has the maximal dimension (i.e., it is pre-Legendrian) and fibers of $\pi$ are connected, $(M, \mathcal{H}, \mathcal{X})$ is a regular completely integrable contact structure studied by Banyaga and Molino [2]. The analysis above lead us to the following definition (Section 5).

Let $X$ be a contact vector field. We shall say that a contact equation

$$
\dot{x}=X
$$

is contact noncommutatively integrable if there is an Abelian Lie algebra of contact symmetries $\mathcal{X}$, an open dense set $M_{\text {reg }} \subset M$, and a submersion $\pi: M_{\text {reg }} \rightarrow W$ such that

(i) The contact vector field $X$ is tangent to the fibers of $\pi$;

(ii) $\left(M_{\text {reg }}, \mathcal{H}, \mathcal{X}\right)$ is a complete pre-isotropic contact structure.

Analogs to the Mishchenko-Fomenko-Nehoroshev theorem, we prove that in a neighborhood of any invariant torus, there exist canonical generalized contact action-angle coordinates and (1.1) is a translation in angle variables, where frequencies depend only on actions and in which the contact distribution $\mathcal{H}$ is presented by the canonical 1-form $\alpha_{0}$ (Theorem 5.1).

For the co-oriented case, we also formulate the statement involving only integrals of a motion (Theorem 5.2): a contact equation (1.1) is noncommutatively integrable if it possesses a collection of first integrals $f_{1}, f_{2}, \ldots, f_{2 n-r}$, that are all in involution with the constant functions and with the first $r$ integrals:

$$
\left[1, f_{i}\right]=0, \quad\left[f_{i}, f_{j}\right]=0, \quad i=1, \ldots, 2 n-r, \quad j=1, \ldots, r .
$$

Note that, besides integrable geodesic flows on homogeneous spaces restricted to the unit co-sphere bundles $[\mathbf{4}, \mathbf{1 6}]$, a natural class of examples of contact flows integrable in a noncommutative sense are the Reeb flows on $K$-contact manifolds $\left(M^{2 n+1}, \alpha\right)$ where the rank of the manifold is less then $n+1$ (see Yamazaki [35] and Lerman [23]).

Finally in Section 6, we consider a complete pre-isotropic contact structure $(M, \alpha, \mathcal{X})$ of the Reeb type, i.e., $\mathcal{H}$ is defined by a global $\mathcal{X}$-invariant form $\alpha$ and the Reeb vector field of $\alpha$ is $\pi$-vertical. Note that $(M, \mathcal{H}, \mathcal{X})$ can be a complete pre-isotropic structure with a global $\mathcal{X}$-invariant contact form $\alpha$, which is not of the Reeb type (see Proposition 6.1). On the other hand, the invariant foliation $\mathcal{F}$ of a complete pre-isotropic contact structure $(M, \alpha, \mathcal{X})$ of the Reeb type is $\alpha$-complete (Proposition 6.2). We describe the transition 
functions between the contact action-angle coordinates (Proposition 6.3) and prove the statement on the existence of global action-action variables in the case when $\pi: M \rightarrow W$ is a trivial principal $\mathbb{T}^{r+1}$-bundle (Theorem 6.1).

\section{Contact manifolds and the Jacobi bracket}

In the definitions and notations, we mostly follow Libermann and Marle [25].

A contact form $\alpha$ on a $(2 n+1)$-dimensional manifold $M$ is a Pfaffian form satisfying $\alpha \wedge(d \alpha)^{n} \neq 0$. By a contact manifold $(M, \mathcal{H})$ we mean a connected $(2 n+1)$-dimensional manifold $M$ equipped with a nonintegrable contact (or horizontal) distribution $\mathcal{H}$, locally defined by a contact form: $\left.\mathcal{H}\right|_{U}=\left.\operatorname{ker} \alpha\right|_{U}, U$ is an open set in $M$.

Two contact forms $\alpha$ and $\alpha^{\prime}$ define the same contact distribution $\mathcal{H}$ on $U$ if and only if $\alpha^{\prime}=a \alpha$ for some nowhere vanishing function $a$ on $U$. The condition $\alpha \wedge(d \alpha)^{n} \neq 0$ implies that the form $\left.d \alpha\right|_{x}$ is nondegenerate (symplectic) structure restricted to $\mathcal{H}_{x}$. The conformal class of $\left.d \alpha\right|_{x}$ is invariant under the change $\alpha^{\prime}=a \alpha$. If $\mathcal{V}$ is a linear subspace of $\mathcal{H}_{x}$, then we have well-defined orthogonal complement $\operatorname{orth}_{\mathcal{H}} \mathcal{V} \subset \mathcal{H}_{x}$ with respect to $\left.d \alpha\right|_{x}$, as well as the notion of the isotropic $\left(\mathcal{V} \subset \operatorname{orth}_{\mathcal{H}} \mathcal{V}\right)$, coisotropic $\left(\mathcal{V} \supset \operatorname{orth}_{\mathcal{H}} \mathcal{V}\right)$ and the Lagrange subspaces $\left(\mathcal{V}=\operatorname{orth}_{\mathcal{H}} \mathcal{V}\right)$ of $\mathcal{H}_{x}$.

A contact diffeomorphism between contact manifolds $(M, \mathcal{H})$ and $\left(M^{\prime}, \mathcal{H}^{\prime}\right)$ is a diffeomorphism $\phi: M \rightarrow M^{\prime}$ such that $\phi_{*} \mathcal{H}=\mathcal{H}^{\prime}$. If a local 1-parameter group of a vector field $X$ is made of contact diffeomorphisms, $X$ is called an infinitesimal automorphism of a contact structure $(M, \mathcal{H})$ or a contact vector field. Locally, if $\mathcal{H}=\operatorname{ker} \alpha$, then $\mathcal{L}_{X} \alpha=\lambda \alpha$, for some smooth function $\lambda$.

The existence of a global contact form $\alpha$ is equivalent to the coorientability of $\mathcal{H}[\mathbf{1 5}]$. From now on we consider a co-oriented (or strictly) contact manifold $(M, \alpha)$. The Reeb vector field $Z$ is a vector field uniquely defined by

$$
i_{Z} \alpha=1, \quad i_{Z} d \alpha=0 .
$$

The tangent bundle $T M$ and the cotangent bundle $T^{*} M$ are decomposed into

$$
T M=\mathcal{Z} \oplus \mathcal{H}, \quad T^{*} M=\mathcal{Z}^{0} \oplus \mathcal{H}^{0},
$$

where $\mathcal{Z}=\mathbb{R} Z$ is the kernel of $d \alpha, \mathcal{Z}^{0}$ and $\mathcal{H}^{0}=\mathbb{R} \alpha$ are the annihilators of $\mathcal{Z}$ and $\mathcal{H}$, respectively. The sections of $\mathcal{Z}^{0}$ are called semi-basic forms.

According to (2.1), we have decompositions of vector fields and 1-forms

$$
X=\left(i_{X} \alpha\right) Z+\hat{X}, \quad \eta=\left(i_{Z} \eta\right) \alpha+\hat{\eta},
$$

where $\hat{X}$ is horizontal and $\hat{\eta}$ is semi-basic.

The mapping $\alpha^{b}: X \mapsto-i_{X} d \alpha$ carries $X$ onto a semi-basic form. The restriction of $\alpha^{b}$ to horizontal vector fields is an isomorphism whose inverse will be denoted by $\alpha^{\sharp}$. The mapping

$$
\Phi: \mathcal{N} \longrightarrow C^{\infty}(M), \quad \Phi(X)=i_{X} \alpha
$$


establish the isomorphism between the vector space $\mathcal{N}$ of infinitesimal contact automorphisms onto the set $C^{\infty}(M)$ of smooth functions on $M$, with the inverse (see $[\mathbf{2 4}, \mathbf{2 5}]$ )

$$
\Phi^{-1}(f)=f Z+\alpha^{\sharp}(\widehat{d f}) .
$$

The vector field $X_{f}=\Phi^{-1}(f)$ is called the contact Hamiltonian vector field and

$$
\dot{x}=X_{f}
$$

contact Hamiltonian equation corresponding to $f$. Note that

$$
\mathcal{L}_{X_{f}} \alpha=d f(Z) \alpha
$$

and $X_{f}$ is an infinitesimal automorphism of $\alpha\left(\mathcal{L}_{X_{f}} \alpha=0\right)$ if and only if $d f$ is semi-basic. Note that $\Phi(Z)=1$, i.e., $Z=X_{1}$.

The mapping (2.3) is a Lie algebra isomorphism, where on $\mathcal{N}$ we have the usual bracket and the Jacobi bracket on $C^{\infty}(M)$ defined by $[f, g]=$ $\Phi\left[X_{f}, X_{g}\right]$ :

$$
X_{[f, g]}=\left[X_{f}, X_{g}\right], \quad X_{[1, f]}=\left[Z, X_{f}\right] .
$$

Note that $d f$ is semi-basic, if and only if $[1, f]=\left[Z, X_{f}\right]=0$.

Together with the Jacobi bracket, we have the associated Jacobi bi-vector field $\Lambda$ :

$$
\Lambda(\eta, \xi)=d \alpha\left(\alpha^{\sharp} \hat{\eta}, \alpha^{\sharp} \hat{\xi}\right) .
$$

Let $\Lambda^{\sharp}: T^{*} M \rightarrow T M$ be the morphism defined by $\left\langle\Lambda_{x}^{\sharp}\left(\eta_{x}\right), \xi_{x}\right\rangle=$ $\Lambda_{x}\left(\eta_{x}, \xi_{x}\right)$, for all $x \in M, \eta_{x}, \xi_{x} \in T_{x}^{*} M$. Then $X_{f}$ may be written as $X_{f}=f Z+\Lambda^{\sharp}(d f)$.

It can be easily checked that

$$
[f, g]=d \alpha\left(X_{f}, X_{g}\right)+f \mathcal{L}_{Z} g-g \mathcal{L}_{Z} f=\Lambda(d f, d g)+f \mathcal{L}_{Z} g-g \mathcal{L}_{Z} f .
$$

The derivation of functions along the contact vector field $X_{f}$ can be described by the use of the Jacobi bracket

$$
\mathcal{L}_{X_{f}} g=[f, g]+g \mathcal{L}_{Z} f .
$$

Thus, if $d f$ and $d g$ are semi-basic, we have the following important property of the Jacobi bracket $[f, g]$.

Lemma 2.1. Suppose that $d f$ and $d g$ are semi-basic. Then

$$
[f, g]=d \alpha\left(X_{f}, X_{g}\right)=\Lambda(d f, d g)
$$

and the following statements are equivalent:

(i) $f$ and $g$ are in involution: $[f, g]=0$, i.e., Hamiltonian contact vector fields $X_{f}$ and $X_{g}$ commute: $\left[X_{f}, X_{g}\right]=0$.

(ii) $g$ is the integral of the contact vector field $X_{f}: \mathcal{L}_{X_{f}} g=0$.

(iii) $f$ is the integral of the contact vector field $X_{g}: \mathcal{L}_{X_{g}} f=0$. 
Moreover, if $\mathcal{Z}$ is a simple foliation, i.e., there exists a surjective submersion $\pi: M \rightarrow P$ and the distribution $\mathcal{Z}$ consist of vertical spaces of the submersion: $\mathcal{Z}=\operatorname{ker} \pi_{*}$, then the base manifold $P$ has a nondegenerate Poisson structure $\{\cdot, \cdot\}$ such that $[f, g]=\pi^{*}\{\bar{f}, \bar{g}\}, f=\bar{f} \circ \pi, g=\bar{g} \circ \pi[\mathbf{6}, 25]$.

\section{3. $\alpha$-Complete pre-isotropic foliations}

Let $\mathcal{F}$ be a foliation on a co-oriented contact manifold $\left(M^{2 n+1}, \alpha\right)$. The pseudo-orthogonal distribution $\mathcal{F}^{\perp}$ is defined by

$$
\mathcal{F}^{\perp}=\mathcal{Z} \oplus \Lambda^{\sharp}\left(\mathcal{F}^{0}\right) \text {. }
$$

where $\mathcal{F}^{0}$ is the annihilator of $\mathcal{F}$. It is locally generated by the Reeb vector field $Z$ and the contact Hamiltonian vector fields, that correspond to the first integrals of $\mathcal{F}$.

A foliation $\mathcal{F}$ is said to be $\alpha$-complete if for any pair $f_{1}, f_{2}$ of first integrals of $\mathcal{F}$ (where $f_{i}$ may be a constant), the bracket $\left[f_{1}, f_{2}\right]$ is also a first integral of $\mathcal{F}$ (eventually a constant).

Theorem 3.1 (Libermann [26]). A foliation $\mathcal{F}$ on $\left(M^{2 n+1}, \alpha\right)$ containing the Reeb vector field $Z$ is $\alpha$-complete if and only if the pseudo-orthogonal subbundle $\mathcal{F}^{\perp}$ is integrable, defining a foliation that is also $\alpha$-complete and $\left(\mathcal{F}^{\perp}\right)^{\perp}=\mathcal{F}$. Then for any pair of integrals $f, g$ of $\mathcal{F}$ and $\mathcal{F}^{\perp}$, respectively, we have $[f, g]=0$.

Let $p$ be the rank of $\mathcal{F}^{0}$ and $f_{1}, \ldots, f_{p}$ be a set of independent integrals of $\mathcal{F}$ in an open set $U$. Since $\operatorname{ker} \Lambda_{x}^{\sharp}=\mathbb{R} \alpha_{x}, \operatorname{dim} \mathcal{F}_{x}^{\perp}$ is equal to $p+1$ or $p$, depending the forms $\alpha, d f_{1}, \ldots, d f_{p}$ are linearly independent or not. In the later case, the form induced by $\alpha$ on the leaf passing through $x$ vanishes at $x$. Conversely, if $\left.\alpha\right|_{\mathcal{F}}=0$, i.e., $\mathcal{F} \subset \mathcal{H}$, then $\operatorname{dim} \mathcal{F}^{\perp}=p$.

A foliation $\mathcal{G}$ is pseudo-isotropic if $\mathcal{G} \subset \mathcal{H}[\mathbf{2 7}] .{ }^{2}$ Then $\alpha$ is a section of $\mathcal{G}^{0}$, the distribution $\mathcal{G}^{\perp}$ has the constant rank $p$ and $\mathcal{G}^{\perp}$ is a vector bundle. A Legendre foliation is a pseudo-isotropic foliation of maximum rank $n$. Then $\operatorname{dim} \mathcal{G}^{0}=\operatorname{dim} \mathcal{G}^{\perp}=n+1$.

By the analogy with a pre-isotropic embedding (see Lerman [22]), we introduce:

Definition 3.1. A foliation $\mathcal{F}$ is pre-isotropic if

(i) $\mathcal{F}$ is transversal to $\mathcal{H}$.

(ii) $\mathcal{G}=\mathcal{F} \cap \mathcal{H}$ is an isotropic subbundle of $\mathcal{H}$.

Lemma 3.1. Condition (ii) is equivalent to the condition that $\mathcal{G}=\mathcal{F} \cap \mathcal{H}$ is a pseudo-isotropic foliation.

\footnotetext{
${ }^{2}$ Submanifolds $G \subset M$ that are integral manifolds of $\mathcal{H}$ are also called isotropic submanifolds, e.g., see [14]. Here we keep Libermann's notation.
} 
Proof. Let $\left(f_{1}, \ldots, f_{p}\right)$ be a set of local integrals of $\mathcal{F}$ and let $X, Y$ be sections of $\mathcal{G}$. Then $\alpha, d f_{1}, \ldots, d f_{p}$ are linearly independent and we have

$$
\begin{aligned}
& d f_{i}(X)=d f_{i}(Y)=\alpha(X)=\alpha(Y)=0, \\
& d f_{i}([X, Y])=\mathcal{L}_{X} \mathcal{L}_{Y} f_{i}-\mathcal{L}_{Y} \mathcal{L}_{X} f_{i}=0, \\
& d \alpha(X, Y)=\mathcal{L}_{X} \alpha(Y)-\mathcal{L}_{Y} \alpha(X)-\alpha([X, Y])=-\alpha([X, Y]) .
\end{aligned}
$$

Therefore, $\mathcal{G}$ is an isotropic subbundle of $\mathcal{H}$ if and only if it is integrable.

Theorem 3.2. Let $\mathcal{F}$ be a pre-isotropic foliation containing the Reeb vector field $Z$.

(i) We have the flag of distributions $(\mathcal{G}, \mathcal{F}, \mathcal{E})$ :

$$
\mathcal{G}=\mathcal{F} \cap \mathcal{H} \subset \mathcal{F} \subset \mathcal{E}=\mathcal{G}^{\perp}=\mathcal{F}^{\perp} .
$$

On the contrary, if $\mathcal{F}$ is a foliation containing the Reeb vector field $Z$ and (3.1) holds, then $\mathcal{F}$ is a pre-isotropic foliation.

(ii) The foliation $\mathcal{F}$ (or $\mathcal{G})$ is $\alpha$-complete, if and only if $\mathcal{E}$ is completely integrable. Assume $\mathcal{E}$ is integrable and let $f_{1}, \ldots, f_{p}$ and $y_{1}, \ldots, y_{r}, 2 n-p=r$ be any sets of local integrals of $\mathcal{F}$ and $\mathcal{E}$, respectively. Then:

$$
\left[f_{i}, y_{j}\right]=0, \quad\left[y_{j}, y_{k}\right]=0, \quad\left[f_{i}, 1\right]=0, \quad\left[y_{i}, 1\right]=0 .
$$

(iii) Each leaf of an $\alpha$-complete pre-isotropic foliation $\mathcal{F}$ as well as each leaf of the corresponding pseudo-isotropic foliation $\mathcal{G}$ has an affine structure.



Figure 1. Illustration of Theorem 3.2: a torus $F$ is a leaf through $x$. 
Proof. (i) Let $\mathcal{G}=\mathcal{F} \cap \mathcal{H}$ be isotropic. We have $\mathcal{G}^{0}=\left\langle\mathcal{F}^{0}, \alpha\right\rangle$ and $\operatorname{ker} \Lambda^{\sharp}=$ $\mathbb{R} \alpha$. Thus:

$$
\begin{aligned}
\mathcal{F}^{\perp}=\mathcal{G}^{\perp} & =\mathcal{Z} \oplus \Lambda^{\sharp}\left(\mathcal{G}^{0}\right)=\mathcal{Z} \oplus \Lambda^{\sharp}\left(\mathcal{G}^{0} \cap \mathcal{Z}^{0}\right) \\
& =\mathcal{Z} \oplus \alpha^{\sharp}\left(\mathcal{G}^{0} \cap \mathcal{Z}^{0}\right)=\mathcal{Z} \oplus \operatorname{orth}_{\mathcal{H}} \mathcal{G} \supset \mathcal{Z} \oplus \mathcal{G}=\mathcal{F} .
\end{aligned}
$$

(ii) This item follows directly from Theorem 1 and the fact that integrals of $\mathcal{E}$ are also integrals of $\mathcal{F}$. Note that $d f_{i}$ and $d y_{j}$ are semi-basic and $X_{f_{i}}$, $X_{y_{j}}$ are infinitesimal automorphisms of $\alpha$.

(iii) Let $U$ be an open set where we have defined commuting integrals $y_{1}, \ldots, y_{r}$ of $\left.\mathcal{E}\right|_{U}$. Since $\mathcal{E}^{\perp}=\mathcal{F}$, the distribution $\left.\mathcal{F}\right|_{U}$ is generated by a contact commuting vector fields $Z, X_{y_{1}}, \ldots, X_{y_{r}}$ :

$$
\left[Z, X_{y_{i}}\right]=0, \quad\left[X_{y_{i}}, X_{y_{j}}\right]=0 .
$$

The distribution $\left.\mathcal{G}\right|_{U}$ is generated by their horizontal parts $\hat{X}_{y_{1}}, \ldots, \hat{X}_{y_{r}}$ which also commute. Indeed, since $\mathcal{G}$ is integrable $\left[\hat{X}_{y_{i}}, \hat{X}_{y_{j}}\right]$ is a section of $\mathcal{G}$, in particular it is horizontal. Further

$$
\begin{aligned}
0= & {\left[X_{y_{i}}, X_{y_{j}}\right]=\left[y_{i} Z+\hat{X}_{y_{i}}, y_{j} Z+\hat{X}_{y_{j}}\right] } \\
= & {\left[y_{i} Z, y_{j} Z\right]+\left[\hat{X}_{y_{i}}, \hat{X}_{y_{j}}\right]+y_{i}\left[Z, \hat{X}_{y_{j}}\right]+y_{j}\left[\hat{X}_{y_{i}}, Z\right] } \\
& -\mathcal{L}_{\hat{X}_{y_{j}}}\left(y_{i}\right) Z+\mathcal{L}_{\hat{X}_{y_{i}}}\left(y_{j}\right) Z .
\end{aligned}
$$

On the other hand, since $\mathcal{L}_{Z} y_{i}=0$, we have

$$
\begin{aligned}
0 & =\left[Z, X_{y_{i}}\right]=\left[Z, y_{i} Z+\hat{X}_{y_{i}}\right]=\left[Z, y_{i} Z\right]+\left[Z, \hat{X}_{y_{i}}\right] \\
& =\mathcal{L}_{Z}\left(y_{i}\right) Z+\left[Z, \hat{X}_{y_{i}}\right]=\left[Z, \hat{X}_{y_{i}}\right] .
\end{aligned}
$$

Therefore, taking the horizontal part in (3.2) we get

$$
\left[\hat{X}_{y_{i}}, \hat{X}_{y_{j}}\right]=0 \text {. }
$$

Thus, locally we have parallelism both on $\mathcal{F}=\left\langle Z, \hat{X}_{y_{1}}, \ldots, \hat{X}_{y_{r}}\right\rangle$ and $\mathcal{G}=\left\langle\hat{X}_{y_{1}}, \ldots, \hat{X}_{y_{r}}\right\rangle$. Now, let $U^{\prime}$ be an open set $\left(U \cap U^{\prime} \neq \emptyset\right)$ and let $y_{1}^{\prime}, \ldots, y_{r}^{\prime}$ be commuting integrals of $\left.\mathcal{E}\right|_{U^{\prime}}$. Then, on $U \cap U^{\prime}$ we have

$$
\begin{aligned}
& y_{i}^{\prime}=\varphi_{i}\left(y_{1}, \ldots, y_{r}\right), \quad i=1, \ldots, r \\
& d y_{i}^{\prime}=\sum_{j} \frac{\partial \varphi_{i}}{\partial y_{j}} d y_{j} .
\end{aligned}
$$

From the definition $\hat{X}_{y_{i}^{\prime}}=\alpha^{\sharp}\left(\widehat{d y_{i}^{\prime}}\right)=\alpha^{\sharp}\left(d y_{i}^{\prime}-\left(i_{Z} d y_{i}^{\prime}\right) \alpha\right)=\alpha^{\sharp}\left(d y_{i}^{\prime}\right)$, we get the fiber-wise linear transformation

$$
\hat{X}_{y_{i}^{\prime}}=\sum_{j} \frac{\partial \varphi_{i}}{\partial y_{j}} \hat{X}_{y_{j}^{\prime}}, \quad i=1, \ldots, r,
$$

which shows that the parallelism of $\mathcal{G}$ and $\mathcal{F}$ is independent of the chart. 
If $\mathcal{F}$ has the maximal dimension $n+1$ then $\mathcal{F}$ is pre-Legendrian, while $\mathcal{G}$ is a Legendrian foliation. The existence of an affine structure is already known for $\alpha$-complete Legendre foliations $[\mathbf{1 9 , 2 6 , 2 7 , 3 2}$. This imposes restrictions on the topology of the leaves. In particular, compact leaves of $\mathcal{G}$ and $\mathcal{F}$ are tori.

Of particular interest is the case when $\mathcal{F}$ is a simple foliation, i.e., the leaves of the foliation are fibers of the submersion. We will study such a situation in the next section.

\section{Complete pre-isotropic contact structures}

In this section, a contact structure does not need to be co-oriented.

Let $(M, \mathcal{H})$ be a $(2 n+1)$-dimensional contact manifold and let

$$
\pi: M \rightarrow W
$$

be a proper submersion on $p$-dimensional manifold $W, p \geq n$. Define the distribution $\mathcal{F}$ as the kernel of $\pi_{*}: T M \rightarrow T W$, i.e., the leaves of $\mathcal{F}$ are fibers of $\pi$.

Definition 4.1. We shall say that $(M, \mathcal{H}, \mathcal{X})$ is a complete pre-isotropic contact structure if

(i) $\mathcal{F}$ is pre-isotropic, i.e., it is transversal to $\mathcal{H}$ and $\mathcal{G}=\mathcal{F} \cap \mathcal{H}$ is an isotropic subbundle of $\mathcal{H}$, or, equivalently $\mathcal{G}$ is a foliation.

(ii) $\mathcal{X}$ is an Abelian Lie algebra of infinitesimal contact automorphisms of $\mathcal{H}$, which has the fibers of $\pi$ as orbits.

In the case $p=n$ (and connected fibers), we have a regular completely integrable contact structure $(M, \mathcal{H}, \mathcal{X})$ studied in Banyaga and Molino [2].

Suppose $\mathcal{F}$ is an $\alpha$-complete foliation with compact leaves (according to the presence of the affine structure, the leaves are tori). Locally, in a neighborhood $U$ of any fixed torus $F$ the foliation is simple. There is a surjective submersion $\pi: U \rightarrow W=U / \mathcal{F}, \mathcal{F}=\operatorname{ker} \pi_{*}$. We can define an Abelian Lie algebra $\mathcal{X}$ of infinitesimal automorphisms of $\mathcal{H}$ by $Z, X_{y_{1}}, \ldots, X_{y_{r}}$ where, $y_{1}, \ldots, y_{r}$ are integrals of $\mathcal{E}=\mathcal{F}^{\perp}$. Thus, we have well-defined complete pre-isotropic contact structure $(U, \mathcal{H}, \mathcal{X})$.

On the contrary, we also have:

Theorem 4.1. Let $(M, \mathcal{H}, \mathcal{X})$ be a complete pre-isotropic contact structure related to the submersion (4.1). Every point of $M$ has an open, $\mathcal{X}$-invariant neighborhood $U$ on which the contact structure can be represented by a local contact form $\alpha_{U}$ such that:

(i) $\alpha_{U}$ is invariant by all elements of $\mathcal{X}$;

(ii) the restriction of $\mathcal{F}$ to $U$ is $\alpha_{U}$-complete.

Proof. (i) The proof of item (i) is a modification of the proof given in [2] for a regular completely integrable contact structure. From the definition, 
for every point $x_{0}$ of $M$, there exist $X \in \mathcal{X}$ transverse to $\mathcal{H}_{x_{0}}$. The vector field $X$ is then transverse to $\mathcal{H}$ in some neighborhood $U_{2}$ of $x_{0}$. Let $\alpha_{0}$ be a contact form defining $\mathcal{H}$ in $U_{1} \subset U_{2}$. Then $\alpha_{0}(X) \neq 0$ on $U_{1}$ and define $\alpha=\alpha_{0} / \alpha_{0}(X)$.

Since $\mathcal{X}$ is Abelian, we have $i_{[Y, X]} \alpha=0, Y \in \mathcal{X}$. Also, $i_{X} \alpha=1$ and $\mathcal{L}_{Y} \alpha=\lambda \alpha$, for some function $\lambda$ defined in $U_{1}$. Thus

$$
0=i_{[Y, X]} \alpha=\mathcal{L}_{Y} i_{X} \alpha-i_{X} \mathcal{L}_{Y} \alpha=\mathcal{L}_{Y} 1-i_{X}(\lambda \alpha)=-\lambda,
$$

i.e., $Y$ is an infinitesimal automorphism of $\alpha$. Since $\alpha$ is invariant by $\mathcal{X}$ and the orbits of $\mathcal{X}$ are the fibers of the submersion (4.1), the form $\alpha$ is well-defined on $U=\pi^{-1}\left(\pi\left(U_{1}\right)\right)$ as well.

(ii) The foliation $\left.\mathcal{F}\right|_{U}$ is $\alpha$-complete if and only if $\left.\mathcal{E}\right|_{U}=\left.\mathcal{F}^{\perp}\right|_{U}$ is an integrable distribution.

From the identity

$$
0=\mathcal{L}_{X} \alpha=i_{X} d \alpha+d i_{X} \alpha=i_{X} d \alpha
$$

we get that $X$ is the Reeb vector field of $\alpha$ on $U$. Denote $Z=X$.

Let $X_{1}, \ldots, X_{r} \in \mathcal{X}$ be vector fields such that $Z, X_{1}, \ldots, X_{r}$ span the foliation $\left.\mathcal{F}\right|_{U}$. Therefore, the corresponding contact Hamiltonians

$$
y_{i}=\Phi\left(X_{i}\right)=i_{X_{i}} \alpha
$$

are independent functions on $U$. Besides, $y_{i}$ are $\pi$-vertical:

$$
0=i_{\left[X, X_{i}\right]} \alpha=\mathcal{L}_{X} i_{X_{i}} \alpha-i_{X_{i}} \mathcal{L}_{X} \alpha=\mathcal{L}_{X} y_{i},
$$

for all $X \in \mathcal{X}$.

The corank of the distribution $\left.\mathcal{E}\right|_{U}$ is $r=2 n-p$. It is integrable and has $y_{1}, \ldots, y_{r}$ as independent integrals. Indeed, by definition we have

$$
\mathcal{E}_{U}=\left\langle X_{f} \mid f=\bar{f} \circ \pi, \bar{f} \in C^{\infty}(\pi(U))\right\rangle .
$$

Since $f=\bar{f} \circ \pi$ and $y_{i}$ are $\pi$-vertical we have, in particular, $\mathcal{L}_{Z} f=\mathcal{L}_{Z} y_{i}=$ 0 (the differential $d f$ and $d y_{i}$ are semi-basic on $U$ ). Now, by using $\mathcal{L}_{X_{y_{i}}} f=0$ and Lemma 2.1 we get

$$
\mathcal{L}_{X_{f}} y_{i}=0, \quad i=1, \ldots, r .
$$

The relations (4.2) and (4.3) prove the claim.

\section{Noncommutative contact integrability}

Let us consider a contact vector field $X$ and a contact equation

$$
\dot{x}=X
$$

on a $(2 n+1)$-dimensional contact manifold $(M, \mathcal{H})$.

First, recall a general definition of non-Hamiltonian integrability (e.g., see $[\mathbf{3}, \mathbf{2 0}, \mathbf{3 6}])$, slightly adopted with respect to the notations above. Equation 
(5.1) is (non-Hamiltonian) completely integrable if there is an open dense subset $M_{\text {reg }} \subset M$ and a proper submersion

$$
\pi: M_{\text {reg }} \rightarrow W
$$

to a $p$-dimensional manifold $W$ and an Abelian Lie algebra $\mathcal{X}$ of symmetries such that:

(i) the contact vector field $X$ is tangent to the fibers of $\pi$;

(ii) the fibers of $\pi$ are orbits of $\mathcal{X}$.

If (5.1) is completely integrable then $M_{\text {reg }}$ is foliated on $(r+1)$-dimensional tori with a quasi-periodic dynamics. In nonholonomic mechanics, usually, an additional time reparametrization is required (e.g., see $[\mathbf{1 2}, \mathbf{1 7}, \mathbf{2 0}]$ ).

However, the above definition does not reflect the underlying contact structure.

Definition 5.1. We shall say that the contact equation (5.1) is noncommutatively contact completely integrable if, in addition, $\left(M_{\mathrm{reg}}, \mathcal{H}, \mathcal{X}\right)$ is a complete pre-isotropic contact structure.

The regularity of the dynamics of integrable contact systems is described in the following statement.

Theorem 5.1. Suppose that equation (5.1) is noncommutatively contact completely integrable by means of the submersion (5.2) and commuting symmetries $\mathcal{X}$. Let $F$ be a connected component of the fiber $\pi^{-1}\left(w_{0}\right)$. Then $F$ is diffeomorphic to a $r+1$-dimensional torus $\mathbb{T}^{r+1}, r=2 n-p$. There exists an open $\mathcal{X}$-invariant neighborhood $U$ of $F$, an $\mathcal{X}$-invariant contact contact form $\alpha$ on $U$ and a diffeomorphism $\phi: U \rightarrow \mathbb{T}^{r+1} \times D$,

$$
\phi(x)=(\theta, y, x)=\left(\theta_{0}, \theta_{1}, \ldots, \theta_{r}, y_{1}, \ldots, y_{r}, x_{1}, \ldots, x_{2 s}\right), \quad s=n-r,
$$

where $D \subset \mathbb{R}^{p}$ is diffeomorphic to $W_{U}=\pi(U)$, such that

(i) $\left.\mathcal{F}\right|_{U}$ is $\alpha$-complete foliation with integrals $y_{1}, \ldots, y_{r}, x_{1}, \ldots, x_{2 s}$, while the integrals of the pseudo-orthogonal foliation $\left.\mathcal{E}\right|_{U}=\left.\mathcal{F}\right|_{U} ^{\perp}$ are $y_{1}, \ldots, y_{r}$.

(ii) $\alpha$ has the following canonical form

$$
\alpha_{0}=\left(\phi^{-1}\right)^{*} \alpha=y_{0} d \theta_{0}+y_{1} d \theta_{1}+\cdots+y_{r} d \theta_{r}+g_{1} d x_{1}+\cdots+g_{2 s} d x_{2 s} \text {, }
$$

where $y_{0}$ is a smooth function of $y$ and $g_{i}$ are functions of $(y, x)$.

(iii) the flow of $X$ on invariant tori is quasi-periodic

$$
\left(\theta_{0}, \theta_{1}, \ldots, \theta_{r}\right) \longmapsto\left(\theta_{0}+t \omega_{0}, \theta_{1}+t \omega_{2}, \ldots, \theta_{r}+t \omega_{r}\right), \quad t \in \mathbb{R},
$$

where frequencies $\omega_{0}, \ldots, \omega_{r}$ depend only on $y$.

Definition 5.2. We refer to local coordinates $(\theta, y)$ stated in Theorem 5.1 as a generalized contact action-angle coordinates. 
In the case when the contact manifold is co-oriented $(\mathcal{H}=\operatorname{ker} \alpha)$ and we have the contact Hamiltonian equation (2.4), it is convenient to formulate noncommutative integrability in terms of the first integrals and the Jacobi bracket as well.

Theorem 5.2. Suppose we have a collection of integrals $f_{1}, f_{2}, \ldots, f_{2 n-r}$ of equation (2.4) with the contact Hamiltonian either $f=f_{1}$ or $f=1$, where:

$$
\left[1, f_{i}\right]=0, \quad\left[f_{i}, f_{j}\right]=0, \quad i=1, \ldots, 2 n-r, \quad j=1, \ldots, r .
$$

Let $F$ be a compact connected component of the level set

$$
\left\{x \mid f_{1}=c_{1}, \ldots, f_{2 n-r}=c_{2 n-r}\right\}
$$

and assume

$$
d f_{1} \wedge \cdots \wedge d f_{2 n-r} \neq 0
$$

on $F$. Then $F$ is diffeomorphic to a $r+1$-dimensional torus $\mathbb{T}^{r+1}$. There exists a neighborhood $U$ of $F$ with local generalized action-angle coordinates (5.3) in which $\alpha$ has the form (5.4) and the dynamics is quasi-periodic (5.5).

Proof. Consider the mapping

$$
\pi=\left(f_{1}, \ldots, f_{2 n-r}\right): \quad M \rightarrow \mathbb{R}^{2 n-r} .
$$

From (5.7) there exists a neighborhood $U$ of $F$ such that $\left.\pi\right|_{U}$ is a proper submersion to $\pi(U)$. Let $\mathcal{F}$ be a foliation with leaves that are fibers of $\pi$. Since $d f_{i}$ are semi-basic 1-forms, (5.7) implies $d f_{1} \wedge \cdots \wedge d f_{2 n-r} \wedge \alpha \neq 0$. Thus, $\mathcal{F}$ is transversal to $\left.\mathcal{H}\right|_{U}$ and the infinitesimal automorphisms of $\alpha$

$$
Z, X_{f_{1}}, \ldots, X_{f_{r}}
$$

are independent in $U$.

Further, from (2.5) and (5.6), we conclude

$$
\begin{aligned}
& {\left[Z, X_{f_{i}}\right]=0, \quad\left[X_{f_{i}}, X_{f_{j}}\right]=0, \quad i=1, \ldots, 2 n-r, \quad j=1, \ldots, r, \quad \mathcal{L}_{X_{f_{i}}} f_{j}=0,} \\
& \mathcal{L}_{Z} f_{i}=0, \quad L_{X_{f_{j}}} f_{i}=0,
\end{aligned}
$$

The relations (5.9) provide that the commuting vector fields (5.8) belong to $\mathcal{F}$. From the dimensional reason, they span $\mathcal{F}$. From $(5.9)$ we also get that $f_{1}, \ldots, f_{r}$ are integrals of the pseudo-orthogonal distribution $\mathcal{E}=\mathcal{F}^{\perp}$. When $\mathcal{E}$ is integrable. On the other hand, $\mathcal{F} \subset \mathcal{E}$ implies that the distribution $\mathcal{G}=\mathcal{F} \cap \mathcal{H}$ is isotropic (item (i) of Theorem 3.2).

Therefore, $\mathcal{F}$ is a complete pre-isotropic foliation with commuting symmetries (5.8). Now, the statement follows from Theorem 5.1.

Proof of Theorem 5.1. Step 1 (local bi-fibrations). Since on each connected component of the fiber $\pi^{-1}\left(w_{0}\right), \mathcal{X}$ induces a transitive action of $\mathbb{R}^{r+1}(r=$ $2 n-p)$, the connected components of $\pi^{-1}\left(w_{0}\right)$ are $r+1$-dimensional tori $\mathbb{T}^{r+1}$ (e.g., see Arnold [1]). 
Let us fix some connected component $F$ of $\pi^{-1}\left(w_{0}\right)$. Consider some $\mathcal{X}$ invariant connected neighborhood $U$ of $F$ and a $\mathcal{X}$-invariant contact form $\alpha$ defining the distribution $\left.\mathcal{H}\right|_{U}=\operatorname{ker} \alpha$ such that the corresponding Reeb vector field $Z$ belongs to $\mathcal{X}$ (see the construction given in Theorem 4.1).

Let $y_{i}^{\prime}=i_{X_{i}} \alpha$ be contact Hamiltonians of $r$ independent contact vector fields $X_{i} \in \mathcal{X},\left.\mathcal{F}\right|_{U}=\left\langle Z, X_{1}, \ldots, X_{r}\right\rangle$. The functions $y_{1}^{\prime}, \ldots, y_{r}^{\prime}$ are then integrals of the pseudo-orthogonal foliation as well (see the proof of Theorem $3)$. They are $\pi$-vertical, and by $\bar{y}_{i}^{\prime}$ we denote the corresponding functions on $W_{U}=\pi(U)$. Locally, for $U$ small enough, the foliation $\left.\mathcal{E}\right|_{U}$ is also a fibration $\rho_{U}$ over an open set $V_{U}$ diffeomorphic to a ball in $\mathbb{R}^{r}$ with local coordinates $\bar{y}^{\prime}=\left(\bar{y}_{1}^{\prime}, \ldots, \bar{y}_{r}^{\prime}\right)$ (the using of $\bar{y}_{i}^{\prime}$ will be clear from the contexts). Therefore, we have a bi-fibration



with pseudo-orthogonal fibers $\left.\mathcal{F}\right|_{U}$ and $\left.\mathcal{E}\right|_{U}$.

Let $\bar{x}=\left(\bar{x}_{1}, \ldots, \bar{x}_{2 s}\right)$ be any collection of independent functions, where $\left(\bar{y}^{\prime}, \bar{x}\right)$ are local coordinates on $W_{U}$. Let $x_{a}=\bar{x}_{a} \circ \pi_{U}, a=1, \ldots, 2 s$. By the use of the methods developed by Arnold [1] , it follows that locally we have a trivial toric fibration $U \cong \mathbb{T}^{r+1} \times W_{U}$ with coordinates

$$
\left(\varphi_{0}, \ldots, \varphi_{r}, y_{1}^{\prime}, \ldots, y_{r}^{\prime}, x_{1}, \ldots, x_{2 s}\right) .
$$

The angular variables $\left(\varphi_{0}, \ldots, \varphi_{r}\right)$ are chosen such that

$$
Y_{\nu}=\partial / \partial \varphi_{\nu}=\sum_{\mu=0}^{r} \Lambda_{\nu \mu} X_{\mu}
$$

where the Reeb vector field $Z$ of $\alpha$ is denoted by $X_{0}$ and the invertible matrix $\left(\Lambda_{\nu \mu}\right) \in G L(r+1)$ depends only on $\left(y^{\prime}, x\right)$.

Step 2 (description of $\alpha$ ). By construction, the functions $y_{j}^{\prime}=i_{X_{j}} \alpha$ are $\rho_{U}$-basic. Since $\mathcal{L}_{X_{j}} \alpha=0$, the 1 -forms

$$
i_{X_{j}} d \alpha=-d\left(\alpha\left(X_{j}\right)\right)=-d y_{j}^{\prime}, \quad j=1, \ldots, r
$$

are also $\rho_{U}$-basic. ${ }^{3}$ Besides, $i_{X_{0}} d \alpha=i_{Z} d \alpha=0$. Therefore

$$
i_{Y_{\nu}} d \alpha=\sum_{\mu=0}^{r} \Lambda_{\nu \mu} i_{X_{\mu}} d \alpha=-\sum_{\mu=0}^{r} \Lambda_{\nu \mu} d y_{\mu}^{\prime}, \quad \nu=0,1, \ldots, r
$$

\footnotetext{
${ }^{3}$ Let $\pi: M \rightarrow P$ be a surjective submersion. A 1 -form $\omega$ is semi-basic if $i_{X} \omega=0$ for all vertical vector fields $X$. It is basic if $\omega=\pi^{*} \mu$, where $\mu$ is a 1-form on $P$. In particular, a basic form is semi-basic as well [25].
} 
are $\rho_{U}$-semi basic 1 -forms. Here $y_{0}^{\prime} \equiv 1$. In particular, $d \alpha$ does not contain the terms with $d \varphi_{\nu} \wedge d \varphi_{\mu}$. So $\alpha$ takes the form

$$
\alpha=\sum_{\nu=0}^{r} y_{\nu} d \varphi_{\nu}+\sum_{i=1}^{r} \tilde{f}_{i} d y_{i}^{\prime}+\sum_{a=1}^{2 s} \tilde{g}_{a} d x_{a},
$$

where $y_{\nu}=y_{\nu}\left(y^{\prime}, x\right), \nu=0, \ldots, r$. Thus, it follows:

$$
i_{Y_{\nu}} d \alpha=-d y_{\nu}+\sum_{i=1}^{r} \frac{\partial \tilde{f}_{i}}{\partial \varphi_{\nu}} d y_{i}^{\prime}+\sum_{a=1}^{2 s} \frac{\partial \tilde{g}_{a}}{\partial \varphi_{\nu}} d x_{a} .
$$

By combining (5.10), (5.12) and the fact that the matrix $\left(\Lambda_{\nu \mu}\right)$ does not depend on $\varphi$, we obtain that $\tilde{f}_{i}$ and $\tilde{g}_{a}$ are linear in angular variables. Since they are periodic in $\varphi_{\nu}$, they only depend on $\left(y^{\prime}, x\right)$ and

$$
i_{Y_{\nu}} d \alpha=-d y_{\nu}
$$

From (5.11) and (5.13) we find the Lie derivatives

$$
\mathcal{L}_{Y_{\nu}} \alpha=i_{Y_{\nu}} d \alpha+d i_{Y_{\nu}} \alpha=-d y_{\nu}+d y_{\nu}=0, \quad \nu=1, \ldots, r
$$

and conclude that $\alpha$ is invariant with respect to the angle coordinates vector fields $\partial / \partial \varphi_{\nu}=Y_{\nu}$.

Now, according to Lemma 5.1, the matrix $\left(\Lambda_{\nu \mu}\right)$ depends only on $y^{\prime}$ variables. Therefore, the 1-forms $i_{Y_{\nu}} d \alpha$ (see (5.10)) as well as the functions $y_{\nu}$ (see (5.13)) are $\rho_{U}$-basic. Note that $y_{\nu}=i_{Y_{\nu}} \alpha$ are contact Hamiltonians of the contact vector fields $Y_{\nu}$.

Among $y_{\nu}$ there are $r$ independent functions at every point in $U$. With eventually shrinking of $U$ and a permutation of indexes, we can assume that $y_{1}, \ldots, y_{r}$ are independent and $y_{0}=y_{0}\left(y_{1}, \ldots, y_{r}\right)$ (i.e., $\bar{y}_{1}, \ldots, \bar{y}_{r}$ are new coordinates on $V_{U}$ ). As a result, the contact form reads

$$
\alpha=\sum_{\nu=0}^{r} y_{\nu} d \varphi_{\nu}+\sum_{i=1}^{r} f_{i}(y, x) d y_{i}+\sum_{a=1}^{2 s} g_{a}(y, x) d x_{a} .
$$

Introducing the new angle variables

$$
\left(\theta_{0}, \theta_{1}, \ldots, \theta_{r}\right)=\left(\varphi_{0}, \varphi_{1}-f_{1}(y, x), \ldots, \varphi_{r}-f_{r}(y, x)\right),
$$

the form (5.14) becomes

$$
\alpha=\sum_{i=0}^{r} y_{i} d \theta_{i}+\sum_{a=1}^{2 s} g_{a}(y, x) d x_{a}+d f,
$$

where $f=f(y, x)=\sum_{i=1}^{r} y_{i} f_{i}(y, x)$ is a $\pi_{U}$-basic function. Due to the translation (5.15), the coordinate vector fields of $\theta$ and $\varphi$ coincide: $\partial / \partial \theta_{\nu}=$ $\partial / \partial \varphi_{\nu}=Y_{\nu}$. 
Step 3 (Moser's deformation, see, e.g., $[\mathbf{2}, \mathbf{1 4}])$. Let

$$
\alpha_{0}=\sum_{\nu=0}^{r} y_{\nu} d \theta_{\nu}+\sum_{a=1}^{2 s} g_{a}(y, x) d x_{a} .
$$

and $Z=X_{0}$ be the Reeb vector field of $\alpha$. It is $\pi_{U}$-vertical and we have

$$
i_{Z} \alpha=i_{Z} \alpha_{0}=1, \quad i_{Z} d \alpha=i_{Z} d \alpha_{0}=0,
$$

implying $\mathcal{L}_{Z} \alpha=\mathcal{L}_{Z} \alpha_{0}=0$.

Following [2], consider the vector field $Y=-f Z$, where $f$ is the $\pi_{U}$-basic function defined above. The flow $\phi_{t}$ of $Y$ is a complete flow that preserves the toric fibration. Define $\alpha_{t}=\alpha_{0}+t d f$. Then we have

$$
\mathcal{L}_{Y} \alpha_{t}=\mathcal{L}_{Y} \alpha_{0}+t \mathcal{L}_{Y} h=\mathcal{L}_{Y} \alpha_{0}=i_{Y} d \alpha_{0}+d\left(i_{Y} \alpha_{0}\right)=-d f=-\partial \alpha_{t} / \partial t .
$$

Thus

$$
\frac{d}{d t}\left(\phi_{t}^{*} \alpha_{t}\right)=\phi_{t}^{*}\left(\mathcal{L}_{Y} \alpha_{t}+\frac{\partial \alpha_{t}}{\partial t}\right)=0
$$

which implies that $\phi_{1}^{*} \alpha_{1}=\phi_{1}^{*} \alpha=\alpha_{0}$. Finally, the required change of variables is $\phi=\phi_{-1}$.

Step 3 (linearization). Since the system is non-Hamiltonian completely integrable, we have a quasi-periodic motion on invariant tori $[\mathbf{3}, \mathbf{3 6}]$. The special form of a linearization, where frequencies only depend on $y_{1}, \ldots, y_{r}$ follows from Lemma 5.1 below.

Remark 5.1. The action functions $y_{\nu}=i_{Y_{\nu}} \alpha$ constructed above have an another interesting interpretation. Let $\gamma_{\nu}(T)$ be a cycle homologous to the trajectories of the field $\partial / \partial \theta_{\nu}$ restricted to any invariant torus $T$ within $U$. Then it follows

$$
\left.y_{\nu}\right|_{T}=\frac{1}{2 \pi} \int_{\gamma_{\nu}(T)} \alpha
$$

Indeed, since $\left.d \alpha\right|_{T}=0$ (the tangent space of $T$ splits into an isotropic horizontal part and $\mathbb{R} Z=\operatorname{ker} d \alpha$ ) the value of the integral (5.16) is the same for all $\gamma_{\nu}(T)$ in the same homology class. Then (5.16) simply follow from (5.4). In the opposite direction, we can use (5.16) as a definition of $y_{\nu}$. By construction, the functions $y_{\nu}$ are $\pi_{U}$-vertical. As in the symplectic case (see Nehoroshev [31]), it can be proved that they are also $\rho_{U}$-vertical.

Remark 5.2. Let $Z=z_{0}(y) Y_{0}+\cdots+z_{r}(y) Y_{r}$ be the local expression of the Reeb vector field. It is uniquely determined from the conditions $i_{Z} \alpha_{0}=1$, $i_{Z} d \alpha_{0}=0$, i.e.,

$$
z_{0} y_{0}+\cdots+z_{r} y_{r}=1, \quad z_{0} d y_{0}+\cdots+z_{r} d y_{r}=0 .
$$

If $z_{0}=0$ at some point $y=\tilde{y}$, then $z_{1} d y_{1}+\cdots+z_{r} d y_{r}=0$ at $\tilde{y}$. Since $d y_{i}$, $i=1, \ldots, r$ are independent 1 -forms, we get $z_{1}=\cdots=z_{r}=0$ at $\tilde{y}$ which 
contradict (5.17). Therefore $z_{0} \neq 0$ on $V_{U}$. Now, by solving (5.17) we get

$$
z_{0}=\frac{1}{y_{1} \frac{\partial y_{0}}{\partial y_{1}}+\cdots+y_{r} \frac{\partial y_{0}}{\partial y_{r}}-y_{0}}, \quad z_{i}=-\frac{1}{z_{0}} \frac{\partial y_{0}}{\partial y_{i}}, \quad i=1, \ldots, r .
$$

Therefore, typically, the flow of the Reeb vector field is quasi-periodic and everywhere dense in invariant tori. Also, typically, the induced pseudoisotropic foliation $\mathcal{G}=\mathcal{F} \cap \mathcal{H}$ has noncompact invariant manifolds.

Remark 5.3. Consider the 1 -form $\gamma=\sum_{a=1}^{2 s} g_{a}(y, x) d x_{a}=\alpha_{0}-\sum_{\nu=0}^{r} y_{\nu} d \theta_{\nu}$. Since $d \alpha_{0}$ has the maximal rank, according to Darboux's theorem [25], there is a coordinate transformation $q_{j}=q_{j}(y, x), p_{j}=p_{j}(y, x), j=1, \ldots, s$ such that $\gamma=p_{1} d q_{1}+\cdots+p_{s} d q_{s}$, i.e.,

$$
\alpha_{0}=y_{0} d \theta_{0}+y_{1} d \theta_{1}+\cdots+y_{r} d \theta_{r}+p_{1} d q_{1}+\cdots+p_{s} d q_{s} .
$$

Lemma 5.1. Let $(M, \mathcal{H}, \mathcal{X})$ be a complete pre-isotropic contact structure and let $U \subset M$ be an $\mathcal{X}$-invariant set endowed with an $\mathcal{X}$-invariant contact form $\alpha$. Suppose

(i) The foliation $\left.\mathcal{F}\right|_{U}=\left.\operatorname{ker} \pi_{*}\right|_{U}$ is $\alpha$-complete and there exist everywhere independent integrals $y_{1}, \ldots, y_{r}: U \rightarrow \mathbb{R}$, of the pseudo-orthogonal foliation $\left.\mathcal{E}\right|_{U}=\left.\mathcal{F}\right|_{U} ^{\perp}$

(ii) Let $X$ be a contact vector field tangent to the fibers of $\pi_{U}$, commuting with $\mathcal{X}$.

Then $X$ can be written as a fiber-wise linear combination

$$
X=f_{0} Z+f_{1} X_{1}+\cdots+f_{r} X_{r},
$$

where functions $f_{0}, \ldots, f_{r}$ depend only on $y, Z$ is the Reeb vector field of $\alpha$ and $X_{i}=X_{y_{i}}$ are contact Hamiltonian vector fields of $y_{i}, i=1, \ldots, r$.

Proof. Under the assumption (i), $Z, X_{1}, \ldots, X_{r}$ are independent vector fields that generate $\alpha$-complete pre-isotropic foliation $\left.\mathcal{F}\right|_{U}$.

Next, we shall prove that $X$ commute with $Z$. Firstly, note that $Z$ commute with $\mathcal{X}{ }^{4}$ Indeed, let $Y \in \mathcal{X}$. We have

$$
\Phi([Y, Z])=i_{[Y, Z]} \alpha=\mathcal{L}_{Y} i_{Z} \alpha-i_{Z} \mathcal{L}_{Y} \alpha=0 .
$$

Since (2.3) is an isomorphism we get $[Y, Z]=0$.

Secondly, note that any $\pi$-vertical vector field $K$ (not need to be contact field) that commute with $\mathcal{X}$, commute with $X$ as well. Indeed, any point in $U$ has a $\pi$-invariant neighborhood $U^{\prime}$ where $K$ can be written as a linear combination $\sum_{\nu=1}^{r} g_{\nu} Y_{\nu}$ where $g_{\nu}$ are $\pi$-basic functions and $\left(Y_{0}, \ldots, Y_{r}\right)$ is a

\footnotetext{
${ }^{4}$ Here we consider slightly more general situation then it is needed for Theorem 5.1, where, by construction of $\alpha, Z$ is already an element of $\mathcal{X}$. However, we shall use the above formulation for a proof of Proposition 6.2.
} 
collection of vector fields in $\mathcal{X}$ that generate $\left.\mathcal{F}\right|_{U}$. Therefore

$$
[X, K]=\sum_{\nu=0}^{r}\left[X, g_{\nu} Y_{\nu}\right]=\sum_{\nu=0}^{r}\left(g_{\nu}\left[X, Y_{\nu}\right]+d g_{\nu}(X) Y_{\nu}\right)=0
$$

From the above considerations it follows that $X$ commute with $Z$. Let $f=i_{X} \alpha$ be the contact Hamiltonian of $X$. Since $\left[Z, X_{f}\right]=0$ we have $[1, f]=0$ and $d f$ is a semi-basic form. Since $X$ is $\pi_{U}$-vertical, we have $\mathcal{L}_{X_{f}} g=0$, where $g$ is any local integral of $\mathcal{F}$. It is clear that $d g$ is semi-basic and applying Lemma 2.1 again, it follows $\mathcal{L}_{X_{g}} f=0$. When $f$ is an integral of the pseudo-orthogonal foliation $\left.\mathcal{E}\right|_{U}$.

Under the assumptions of Lemma 5.1, integrals of $\left.\mathcal{E}\right|_{U}$ are functions of $y$ and we have $f=f(y)$. Let $f_{i}=\partial f / \partial y_{i}, i=1, \ldots, r$. The forms $d f, d y_{1}, \ldots, d y_{r}$ are semi-basic, so

$$
\begin{aligned}
X & =\Phi^{-1}(f)=f Z+\alpha^{\sharp}(d f)=f Z+\sum_{i=1}^{r} f_{i} \alpha^{\sharp}\left(d y_{i}\right) \\
& =f Z+\sum_{i=1}^{r} f_{i}\left(X_{i}-y_{i} Z\right)=f_{0} Z+f_{1} X_{1}+\cdots+f_{r} X_{r},
\end{aligned}
$$

where $f_{0}=f-\left(y_{1} f_{1}+\cdots+y_{r} f_{r}\right)$.

Remark 5.4. Let $X$ be $\pi_{U}$-horizontal contact vector field. From the proof of the lemma, we see that commuting of $X$ with $\mathcal{X}$ is equivalent to the commuting with the Reeb vector field $Z$, i.e., with the condition that $X$ is an infinitesimal automorphisms of $\alpha$. Also, the condition that $\left.\mathcal{F}\right|_{U}$ is $\alpha$-complete is equivalent to the condition that $Z$ is a section of $\left.\mathcal{F}\right|_{U}$, see Proposition 6.2 given below.

5.1. Discrete systems. Khesin and Tabachnikov defined integrability of discrete

$$
\Psi: M \rightarrow M,
$$

and continuous contact systems (5.1) in terms of the existence of an invariant complete pre-Legendrian foliation $\mathcal{F}$, with additional property that on every leaf $F$ of $\mathcal{F}$, the foliation $\left.\mathcal{G}\right|_{F}$ has a holonomy invariant transverse smooth measure. It turns out that this condition implies the existence of a global contact form $\alpha$ and that $\mathcal{G}$ is an $\alpha$-complete Legendrian foliation [19].

As in [19], we can say that a discrete contact system (5.1) that preserves the contact form $\alpha$ is integrable in a noncommutative sense if it possesses an $\alpha$-complete pre-isotropic invariant foliation $\mathcal{F}$. Also, following the lines of the proof of Lemma $3.5[\mathbf{1 9}]$, one can prove that $\alpha$ determines a holonomy invariant transverse smooth measure of the foliation $\mathcal{G}=\mathcal{F} \cap \mathcal{H}$ restricted to the leaves of $\mathcal{F}$. 
5.2. Examples. For $s=0$, Theorem 4 recover contact action-angle coordinates given by Banyaga and Molino [2]. If $M$ is a compact manifold with a regular effective contact action of $\mathbb{T}^{n+1}$, then $W$ is the sphere $S^{n}$ and for $n \geq 3, M$ is diffeomorphic to $\mathbb{T}^{n+1} \times S^{n}$ (see Lutz $[\mathbf{2 8}]$ ).

Besides noncommutatively integrable geodesic flow restricted to the unit co-sphere bundles $[\mathbf{4}, \mathbf{1 6}]$, a natural class of examples of contact flows integrable in a noncommutative sense are the Reeb flows on $K$-contact manifolds $\left(M^{2 n+1}, \alpha\right)$ where the rank of the manifold is less then $n+1$ (see Yamazaki $[\mathbf{3 5}]$ and Lerman $[\mathbf{2 3}])$.

The regular and almost regular contact manifolds studied by Boothby and Wang $[\mathbf{6}]$ and Thomas $[\mathbf{3 3}]$ provide the most degenerate examples with $\operatorname{dim} W=\operatorname{dim} M-1$.

The billiard system within an ellipsoid in the Euclidean space $\mathbb{R}^{n}$ is one of the basic examples of integrable mappings (e.g., see $[\mathbf{9}, \mathbf{3 4}]$ ). Similarly, the billiard system inside an ellipsoid in the pseudo-Euclidean space $\mathbb{R}^{k, n-k}$ is completely integrable as well. Here, the billiard system is described by a symplectic transformation on the spaces of space-like and time-like geodesics, while it is a contact transformation on the space of light-like geodesics (for more details, see Khesin and Tabachnikov $[\mathbf{1 8}, \mathbf{1 9}]$ ). The considered billiard systems are defined within ellipsoids with different semi-axis. Further properties of ellipsoidal billiards in the pseudo-Euclidean spaces have been studied in $[\mathbf{1 0}]$, where description of periodical trajectories has been derived, including the cases of symmetric ellipsoids. It can be proved that the billiard systems, both in $\mathbb{R}^{n}$ and $\mathbb{R}^{k, n-k}$, within symmetric ellipsoids are completely integrable in the noncommutative sense (the geodesic flow on a symmetric ellipsoid is considered in $[\mathbf{8}]$ ). In particular, the billiard maps restricted to the space of null geodesics are noncommutatively completely integrable contact transformations.

\section{Complete pre-isotropic structures of the Reeb type}

In this section, we consider some global properties of the fibration (4.1).

Proposition 6.1. Let $(M, \mathcal{H}, \mathcal{X})$ be a complete pre-isotropic contact structure and assume that $\mathcal{H}$ is co-oriented. Then there exists a global contact form $\alpha$ representing $\mathcal{H}$ and invariant by elements of $\mathcal{X}$.

Proof. We can cover $W$ by open sets $W_{i}$ such that we have contact 1-forms $\alpha_{U_{i}}$ invariant by $\mathcal{X}$ on every $U_{i}=\pi^{-1}\left(W_{i}\right)$ (Theorem 3 ). Let $\bar{\lambda}_{i}$ be the partition of unity subordinate to covering $\left\{W_{i}\right\}$. Since $\mathcal{H}$ is oriented, for all nonempty intersections $U_{i} \cap U_{j}$, we have smooth positive functions $a_{i j}$, $\alpha_{U_{i}}=\left.f_{i j} \alpha_{U_{j}}\right|_{U_{i} \cap U_{j}}$.

Define the 1 -form $\alpha$ by $\alpha=\sum_{i} \lambda_{i} \alpha_{U_{i}}, \lambda_{i}=\bar{\lambda}_{i} \circ \pi$. Then, on $U_{k}$ we have

$$
\alpha=a_{k} \alpha_{U_{k}}
$$


where $a_{k}=\sum_{i, U_{i} \cap U_{k} \neq \emptyset} \lambda_{i} f_{k i}>0$ is a $\pi$-basic function. When $\alpha$ is a contact form that define $\mathcal{H}$.

It remains to prove $\mathcal{X}$-invariance of $\alpha$. Let $X \in \mathcal{X}$. Then $\mathcal{L}_{X} \lambda_{i}=0$. Further, by construction, $X$ preserve all local contact forms $\alpha_{U_{i}}$. Thus

$$
\mathcal{L}_{X} \alpha=\sum_{i}\left(\mathcal{L}_{X} \lambda_{i}\right) \alpha_{U_{i}}+\lambda_{i} \mathcal{L}_{X} \alpha_{U_{i}}=0
$$

Let $Z$ be the Reeb vector field of the globally $\mathcal{X}$-invariant contact form $\alpha$. Then, as in (5.18), we get $[Z, Y]=0, Y \in \mathcal{X}$. However, it turns out that the foliation $\mathcal{F}=\operatorname{ker} \pi_{*}$ not need to be $\alpha$-complete since $Z$ not need be a section of $\mathcal{F}$.

Recall that a contact toric action on a co-oriented contact manifold $(M, \alpha)$ is of the Reeb type if the Reeb vector field corresponds to an element of the Lie algebra of the torus [5]. Similarly, we give the following definition.

Definition 6.1. Let $(M, \alpha)$ be a co-oriented contact manifold with a complete pre-isotropic contact structure defined by commuting infinitesimal automorphisms $\mathcal{X}$ of $\alpha$, such that the associated Reeb vector field $Z$ is a section of $\mathcal{F}=\operatorname{ker} \pi_{*}$. We refer to a triple $(M, \alpha, \mathcal{X})$ with the above property as a complete pre-isotropic structure of the Reeb type.

Proposition 6.2. Let $(M, \alpha, \mathcal{X})$ be a complete pre-isotropic structure of the Reeb type. Then the associated foliation $\mathcal{F}=\operatorname{ker} \pi_{*}$ is $\alpha$-complete.

Proof. Locally, every leaf $F$ of $\mathcal{F}$ has a $\pi$-invariant neighborhood $U$ with local generalized contact action-angle coordinates (5.3) in which $\mathcal{H}$ is represented by the contact form $\alpha_{0}=\sum_{\nu} y_{\nu} d \theta_{\nu}+\sum_{a} g(y, x) d x_{a}$ and $\left.\mathcal{F}\right|_{U}$ is $\alpha_{0}$-complete (Theorem 5.1). We need to prove that $\mathcal{F}$ is complete with respect to the contact form $\alpha$ as well.

We have $\left.\alpha\right|_{U}=\frac{1}{a} \cdot \alpha_{0}$ for some nonvanishing function $a: U \rightarrow \mathbb{R}$. In what follows, by $Z^{\alpha}, Z^{\alpha_{0}}, X_{f}^{\alpha}, X_{f}^{\alpha_{0}}$ and $\Phi_{\alpha}, \Phi_{\alpha_{0}}$ we denote the Reeb vector fields, contact Hamiltonian vector fields and the isomorphisms (2.3) with respect to $\alpha$ and $\alpha_{0}$, respectively. They are related by

$$
X_{f}^{\alpha}=\Phi_{\alpha}^{-1}(f)=\Phi_{\alpha_{0}}^{-1}(a f)=X_{a f}^{\alpha_{0}}, \quad Z^{\alpha}=\Phi_{\alpha}^{-1}(1)=\Phi_{\alpha_{0}}^{-1}(a)=X_{a}^{\alpha_{0}}
$$

(see Proposition 13.7, [25]).

On the other hand, by the argument used in (5.18), with $\Phi$ replaced by $\Phi_{\alpha}$, we get $\left[Z^{\alpha}, X\right]=0, X \in \mathcal{X}$. Therefore, we can apply Lemma 5.1 with $Z^{\alpha}=X_{a}^{\alpha_{0}}$ and $\alpha_{0}$, instead of $X$ and $\alpha$, concluding that $a$ is a function of actions variables $y=\left(y_{1}, \ldots, y_{r}\right)$ only. 
Let $f$ be an integral of $\mathcal{F}$. Since $d a$ and $d f$ are semi-basic, we get that the contact Hamiltonian vector field

$$
\begin{aligned}
X_{f}^{\alpha} & =\Phi_{\alpha}^{-1}(f)=\Phi_{\alpha_{0}}^{-1}(a f) \\
& =(a f) Z^{\alpha_{0}}+\alpha_{0}^{\sharp}(a d f+f d a)=(a f) Z^{\alpha_{0}}+a \alpha_{0}^{\sharp}(d f)+f \alpha_{0}^{\sharp}(d a) \\
& =a f Z^{\alpha_{0}}+a\left(X_{f}^{\alpha_{0}}-f Z^{\alpha_{0}}\right)+f\left(X_{a}^{\alpha_{0}}-a Z^{\alpha_{0}}\right) \\
& =a X_{f}^{\alpha_{0}}+f Z^{\alpha}-a f Z^{\alpha_{0}},
\end{aligned}
$$

is a section of pseudo-orthogonal complement of $\mathcal{F}$ with respect to $\alpha_{0}$. Thus, the pseudo-orthogonal complements of $\mathcal{F}$ with respect to $\alpha$ and $\alpha_{0}$ coincides. This completeness the proof.

Remark 6.1. Let us return to the construction of an invariant contact form $\alpha$ given in Proposition 6.1. From the proof of Proposition 6.2, we obtain that $\mathcal{F}=\operatorname{ker} \pi_{*}$ is $\alpha$-complete if the functions $a_{k}$ defined by (6.1) depend only on actions variables. If this is not the case, suppose additionally that the Reeb vector field $Z$ is transversal to $\mathcal{F}$ at every point. Then we can consider the foliation $\tilde{\mathcal{F}}$ generated by $\mathcal{X}$ and $Z$. It can be proved that $\tilde{\mathcal{F}}$ is $\alpha$-complete. Note that if $n=p$, i.e., $(M, \mathcal{H}, \mathcal{X})$ is a regular completely integrable contact structure, then $a_{k}$ depends only on action variables and $\mathcal{F}$ is $\alpha$-complete.

Let $(M, \alpha, \mathcal{X})$ be a complete pre-isotropic structure of the Reeb type and assume the fibers of (4.1) are connected. Theorem 5.1 and Proposition 6.2 provide that $\pi: M \rightarrow W$ is a toric fibration. There is an open covering $W_{i}$ of $W$ and local trivializations $\phi_{i}: U_{i}=\pi^{-1}\left(W_{i}\right) \rightarrow \mathbb{T}^{r+1} \times D_{i}$,

$$
\phi_{i}(x)=\left(\theta^{i}, y^{i}, x^{i}\right)=\left(\theta_{0}^{i}, \theta_{1}^{i}, \ldots, \theta_{r}^{i}, y_{1}^{i}, \ldots, y_{r}^{i}, x_{1}^{i}, \ldots, x_{2 s}^{i}\right), \quad s=n-r
$$

where $D_{i} \subset \mathbb{R}^{p}$ is an open set diffeomorphic to $W_{i}$, such that

(i) the fibers of $\pi$ are represented as the level sets of functions $\left(y^{i}, x^{i}\right)$, where the action variables $y^{i}$ are integrals of the pseudo-orthogonal foliation $\mathcal{E}=\mathcal{F}^{\perp}$ restricted to $U_{i}$;

(ii) $\alpha$ has the following canonical form:

$$
\alpha_{i}=\left(\phi_{i}^{-1}\right)^{*} \alpha=y_{0}^{i} d \theta_{0}^{i}+y_{1}^{i} d \theta_{1}^{i}+\cdots+y_{r}^{i} d \theta_{r}^{i}+g_{1}^{i} d x_{1}^{i}+\cdots+g_{2 s}^{i} d x_{2 s}^{i},
$$

where $y_{0}^{i}$ is a smooth function of $y^{i}$ and $g_{a}^{i}$ are functions of $\left(y^{i}, x^{i}\right)$. 
Proposition 6.3. Suppose that the intersection of $W_{i}$ and $W_{j}$, i.e., of $U_{i}$ and $U_{j}$ is connected. Then on $U_{i} \cap U_{j}$ we have the following transition formulas:

$$
\begin{aligned}
& \theta_{\nu}^{j}=\sum_{\mu=0}^{r} M_{\nu \mu}^{i j}\left(\theta_{\mu}^{i}+F_{\mu}^{i j}\left(y^{i}, x^{i}\right)\right), \\
& y_{\nu}^{j}=\sum_{\mu=0}^{r} K_{\nu \mu}^{i j} y_{\mu}^{i}, \quad \nu=0, \ldots, r, \\
& x_{a}^{j}=X_{a}^{i j}\left(y^{i}, x^{i}\right), \quad a=1, \ldots, 2 s,
\end{aligned}
$$

where matrixes $K^{i j}=\left(K_{\nu \mu}^{i j}\right)$ and $M^{i j}=\left(K_{\nu \mu}^{i j}\right)$ belong to $G L(r+1, \mathbb{Z})$, $M=\left(K^{T}\right)^{-1}$, and functions $X_{a}^{i j}\left(y^{i}, x^{i}\right), F_{\nu}^{i j}\left(y^{i}, x^{i}\right)$ satisfy

$$
g_{a}^{i}=\sum_{b=1}^{2 s} g_{b}^{j} \frac{\partial X_{b}^{i j}}{\partial x_{a}^{i}}, \quad \sum_{b=1}^{2 s} g_{b}^{j} \frac{\partial X_{b}^{i j}}{\partial y_{k}^{i}}+\sum_{\nu=0}^{r} y_{\nu}^{i} \frac{\partial F_{\nu}^{i j}}{\partial y_{k}^{i}}=0 .
$$

Proof. Since $y^{i}$ and $y^{j}$ (respectively, $\left(y^{i}, x^{i}\right)$ and $\left.\left(y^{j}, x^{j}\right)\right)$ are integrals of the pseudo-orthogonal foliation $\mathcal{E}$ (respectively, of $\mathcal{F}$ ) we have:

$$
\theta_{\nu}^{j}=\Theta_{\nu}^{i j}\left(\theta^{i}, y^{i}, x^{i}\right), \quad y_{k}^{j}=Y_{k}^{i j}\left(y^{i}\right), \quad x_{a}^{j}=X_{a}^{i j}\left(y^{i}, x^{i}\right),
$$

$\nu=0, \ldots, r, k=1, \ldots, r, a=1 \ldots, 2 s$.

Let us fix some invariant torus $T=\pi^{-1}\left(w_{0}\right)$ within $U_{i} \cap U_{j}\left(w_{0} \in W_{i} \cap\right.$ $W_{j}$ ). From (5.16), we have

$$
\left.y_{\nu}^{j}\right|_{T}=\int_{\gamma_{\nu}^{j}(T)} \alpha=\sum_{\mu=0}^{r} K_{\nu \mu}^{i j} \int_{\gamma_{\mu}^{i}(T)} \alpha=\left.\sum_{\mu=0}^{r} K_{\nu \mu}^{i j} y_{\mu}^{i}\right|_{T},
$$

where $K^{i j} \in G L(r+1, \mathbb{Z})$ is a matrix which relates two different bases of cycles $\left(\gamma_{0}^{j}(T), \ldots, \gamma_{r}^{j}(T)\right)$ and $\left(\gamma_{0}^{i}(T), \ldots, \gamma_{r}^{i}(T)\right)$ defined in Remark 5.1. From (6.6) and the connectedness of $W_{i} \cap W_{j}$ the matrix $K^{i j}$ is constant. This proves (6.3). Therefore

$$
i_{\partial / \partial \theta_{\nu}^{j}} d \alpha=-d y_{\nu}^{j}=-\sum_{\mu} K_{\nu \mu}^{i j} d y_{\mu}^{i}=\sum_{\mu} K_{\nu \mu}^{i j} i_{\partial / \partial \theta_{\mu}^{i}} d \alpha,
$$

implying that $\partial / \partial \theta_{\nu}^{j}-\sum_{\mu} K_{\nu \mu}^{i j} \partial / \partial \theta_{\mu}^{i} \in \operatorname{ker} d \alpha=\mathbb{R} Z$.

Let $\lambda Z$ be the difference of $\partial / \partial \theta_{\nu}^{j}$ and $\sum_{\mu} K_{\nu \mu}^{i j} \partial / \partial \theta_{\mu}^{i}$. Then

$$
\lambda=\alpha(\lambda Z)=\alpha\left(\partial / \partial \theta_{\nu}^{j}-\sum_{\mu} K_{\nu \mu}^{i j} \partial / \partial \theta_{\mu}^{i}\right)=y_{\nu}^{j}-\sum_{\mu} K_{\nu \mu}^{i j} y_{\mu}^{i}=0 .
$$

Thus, from (6.6), permuting the indexes $i$ and $j$, we obtain

$$
\frac{\partial}{\partial \theta_{\nu}^{j}}=\sum_{\mu} \frac{\partial \Theta_{\mu}^{j i}}{\partial \theta_{\nu}^{j}} \frac{\partial}{\partial \theta_{\mu}^{i}}=\sum_{\mu} K_{\nu \mu}^{i j} \frac{\partial}{\partial \theta_{\mu}^{i}},
$$


leading to the fact that $\Theta_{\mu}^{j i}$ is linear in $\theta_{\nu}^{j}$ and that can be written into a form

$$
\Theta_{\mu}^{j i}=\sum_{\nu}\left(K_{\nu \mu}^{i j} \theta_{\nu}^{j}+F_{\nu}^{j i}\left(y^{j}, x^{j}\right)\right) .
$$

From the above expression we get (6.2), where $\sum_{\lambda=0}^{r} K_{\lambda \mu}^{i j} M_{\lambda \nu}^{i j}=\delta_{\nu \mu}$.

Replacing (6.3) and the differentials of (6.2), (6.4) into the identity

$$
\sum_{\nu=0}^{r} y_{\nu}^{i} d \theta_{\nu}^{i}+\sum_{a=1}^{2 s} g_{a}^{i}\left(y^{i}, x^{i}\right) d x_{a}^{i}=\sum_{\lambda=0}^{r} y_{\lambda}^{j} d \theta_{\lambda}^{j}+\sum_{b=1}^{2 s} g_{b}^{j}\left(y^{j}, x^{j}\right) d x_{b}^{j},
$$

and comparing the terms with $d x_{a}^{i}$ and $d y_{k}^{i}$ we get (6.5).

The study of toric fibrations within the symplectic geometry framework is based on the papers of Duistermaat [11] (Lagrangian fibration) and Dazord and Delzant [7] (isotropic fibrations). On the other side, Banyaga and Molino defined characteristic invariants of regular and singular completely integrable contact structures and proved a classification theorem: two completely integrable contact structures with the same invariants are isomorphic [2]. For contact toric actions and singular completely integrable contact structures, see also $[\mathbf{5}, \mathbf{2 2}, \mathbf{2 9}]$, respectively.

Here, we consider the existence of global contact action-angle coordinates by using the arguments already used in the paper.

The possibility of taking all matrices $K^{i j}$ and $M^{i j}$ equal to the identity reflects the fact that the fibration by the invariant tori is a principal $\mathbb{T}^{r+1}$-bundle. When this does not happen, it is said that we have nontrivial monodromy [11].

Let $W^{\prime} \subset W, \operatorname{dim} W^{\prime}=\operatorname{dim} W$ be a connected compact submanifold (with a smooth boundary) and consider the fibration $\pi: M^{\prime} \rightarrow W^{\prime}, M^{\prime}=$ $\pi^{-1}\left(W^{\prime}\right)$. It is obvious that the necessary condition for the existence of global contact action-angle variables is that $M^{\prime} \rightarrow W^{\prime}$ is a trivial principal bundle.

The following sufficient, but not necessary, conditions for $M^{\prime} \rightarrow W^{\prime}$ to be trivial are well known (e.g., see $[\mathbf{1 3}]$ ):

(i) If $W^{\prime}$ is simply connected then $\pi: M^{\prime} \rightarrow W^{\prime}$ is a principal $\mathbb{T}^{r+1}$ bundle.

(ii) In addition, if the second cohomology group $H^{2}\left(W^{\prime}, \mathbb{Z}\right)$ vanish then the principal bundle is trivial and $M^{\prime}$ is diffeomorphic to $\mathbb{T}^{r+1} \times W^{\prime}$.

Indeed, if $W^{\prime}$ is simply connected then the monodromy of the restricted fibration $\pi: M^{\prime} \rightarrow W^{\prime}$ is trivial providing that $\pi: M^{\prime} \rightarrow W^{\prime}$ is a principal $\mathbb{T}^{r+1}$ bundle. For the second assertion, note that the Chern class of $\mathbb{T}^{r+1}=$ $U(1) \times \cdots U(1)$-bundle is equal to

$$
c=c\left(L_{0} \oplus \cdots \oplus L_{r}\right)=\left(1+c_{1}\left(L_{0}\right)\right) \cdots\left(1+c_{1}\left(L_{r}\right)\right),
$$

where $L_{\nu}$ is the bundle associated to the $\nu$ th factor $U(1)$. They are all trivial in the case $H^{2}(M, \mathbb{Z})=0$. When, $\mathbb{T}^{r+1}$-bundle is also trivial. 
Now we can formulate the following statement.

Theorem 6.1 (Global contact action-angle variables). Let $(M, \alpha, \mathcal{X})$ be a complete pre-isotropic structure of the Reeb type and let $W^{\prime} \subset W$, $\operatorname{dim} W^{\prime}=\operatorname{dim} W$ be a connected compact submanifold (with a smooth boundary) such that

(i) $\pi: M^{\prime} \rightarrow W^{\prime}$ is a trivial principal $\mathbb{T}^{r+1}$ bundle, $M^{\prime}=\pi^{-1}\left(W^{\prime}\right)$.

(ii) There exist everywhere independent functions $\bar{x}_{1}, \ldots, \bar{x}_{2 s}$ defined is some neighborhood of $W^{\prime}$ satisfying:

$$
\left\langle d x_{1}, \ldots, d x_{2 s}\right\rangle \cap \mathcal{E}^{0}=0,
$$

where $x_{a}=\bar{x}_{a} \circ \pi$ and $\mathcal{E}=\mathcal{F}^{\perp}$ is the pseudo-orthogonal foliation of $\mathcal{F}$.

Then there exist global action-angle variables $\left(\theta_{0}, \ldots, \theta_{r}, y_{0}, \ldots, y_{r}\right)$ and functions $\bar{g}_{1}, \ldots, \bar{g}_{2 s}: W^{\prime} \rightarrow \mathbb{R}$ such that the contact form $\alpha$ on $M^{\prime}$ reads

$$
\alpha_{0}=y_{0} d \theta_{0}+\cdots+y_{r} d \theta_{r}+\pi^{*}\left(\bar{g}_{1} d \bar{x}_{1}+\cdots+\bar{g}_{2 s} d \bar{x}_{2 s}\right) .
$$

Remark 6.2. Proposition 3 and Theorem 6 are contact analogs of Proposition 1 and Theorem 2' in Nehoroshev [31], respectively. In Theorem 2' [31], instead of the condition (i), the condition that $W^{\prime}$ is a simply connected manifold with vanishing of the second cohomology class $H^{2}\left(W^{\prime}, \mathbb{R}\right)$ is used. A variant of the statement with noncompact invariant manifolds is proved in $[\mathbf{1 3}]$.

Proof. Since $\pi: M^{\prime} \rightarrow W^{\prime}$ is a trivial principal $\mathbb{T}^{r+1}$ bundle, there exist global angles variables $\left(\varphi_{1}, \ldots, \varphi_{r}\right)$. Repeating the arguments used in the proof of Theorem 5.1, we get that the coordinate vector fields $Y_{\nu}=\partial / \partial \varphi_{\nu}$ preserve $\alpha$ and we can define actions as their contact Hamiltonians:

$$
y_{\nu}=\Phi\left(Y_{\nu}\right)=i_{Y_{\nu}} \alpha: \quad M^{\prime} \rightarrow \mathbb{R}, \quad \nu=0, \ldots, r .
$$

They are redundant integrals of the pseudo-orthogonal foliation that satisfy relations (5.17), where $z_{\nu}$ are the components of the Reeb vector field $Z$ with respect to vector fields $Y_{\nu}$. The functions $y_{\nu}$ are $\pi$-basic and let $\bar{y}_{\nu}$ be the corresponding functions on $W^{\prime}, y_{\nu}=\bar{y}_{\nu} \circ \pi$. They are subjected to the constrains

$$
\bar{z}_{0} \bar{y}_{0}+\cdots+\bar{z}_{r} y_{r}=1, \quad \bar{z}_{0} d \bar{y}_{0}+\cdots+\bar{z}_{r} d \bar{y}_{y}=0,
$$

where $z_{\nu}=\pi \circ \bar{z}_{\nu}$.

Moreover, according to the assumption (6.8), in a neighborhood of any point $w_{0} \in W^{\prime}$, we can take $r$ independent functions among $\bar{y}_{\nu}$ that are independent of $\bar{x}_{1}, \ldots, \bar{x}_{2 s}$ providing a local coordinate chart.

Let $\left\{W_{i}\right\}$ be a finite covering of $W^{\prime}$ such that on every $W_{i}$ we can take local coordinates $\left(\bar{y}^{i}, \bar{x}\right)$, where $\left(\bar{y}_{1}^{i}, \ldots, \bar{y}_{r}^{i}\right)$ is a subcollection of redundant actions $\left(\bar{y}_{0}, \ldots, \bar{y}_{r}\right)$.

As in Theorem 5.1 we get that the contact form in $U_{i}=\pi^{-1}\left(W_{i}\right)$ reads

$$
\alpha^{i}=\alpha_{\theta}+\pi^{*} \alpha_{y}^{i}+\pi^{*} \alpha_{x}^{i},
$$


where $\alpha_{\theta}=\sum_{\nu=0}^{r} y_{\nu} d \varphi_{\nu}, \alpha_{y}^{i}=\sum_{k=1}^{r} \bar{f}_{k}^{i}\left(\bar{y}^{i}, \bar{x}\right) d \bar{y}_{k}^{i}, \alpha_{x}^{i}=\sum_{a=1}^{2 s} \bar{g}_{a}^{i}\left(\bar{y}^{i}, \bar{x}\right) d \bar{x}_{a}$.

Thus, on $M^{\prime}$ we have a unique decomposition $\alpha=\alpha_{\theta}+\pi^{*} \alpha_{y}+\pi^{*} \alpha_{x}$, locally given by (6.11). It is obvious that we can write $\alpha_{x}$ as $\alpha_{x}=\sum_{a=1}^{2 s} \bar{g}_{a} d \bar{x}_{a}$, where $\bar{g}_{a}: W^{\prime} \rightarrow \mathbb{R}$.

Next, consider the filtration

$$
V_{1}=W_{1} \subset V_{2}=W_{1} \cup W_{2} \subset \cdots \subset V_{N}=W_{1} \cup \cdots \cup W_{N}=W^{\prime} .
$$

Applying Lemma 6.1 given below $(N-1)$ times we obtain functions $\bar{f}_{0}, \ldots, \bar{f}_{r}: W^{\prime} \rightarrow \mathbb{R}$, satisfying the identities

$$
\bar{f}_{0} d \bar{y}_{0}+\bar{f}_{1} d \bar{y}_{1}+\cdots+\bar{f}_{r} d \bar{y}_{r}=\bar{f}_{1}^{i} d \bar{y}_{1}^{i}+\ldots \bar{f}_{r} d \bar{y}_{r}^{i}
$$

on every $W_{i}$.

Therefore, after globally defined transformation

$$
\left(\theta_{0}, \theta_{1}, \ldots, \theta_{r}\right)=\left(\varphi_{0}-f_{0}, \varphi_{1}-f_{1}, \ldots, \varphi_{r}-f_{r}\right), \quad f_{\nu}=\bar{f}_{\nu} \circ \pi,
$$

the form $\alpha$ becomes

$$
\alpha=\sum_{\nu=0}^{r} y_{\nu} d \theta_{\nu}+\pi^{*} \sum_{a=1}^{2 s} \bar{g}_{a} d \bar{x}_{a}+d f,
$$

where $f=\sum_{\nu} y_{\nu} f_{\nu}$ is a $\pi$-basic function. Now, as in Theorem 5.1, applying Moser's deformation for a compact manifold $M^{\prime}$ and family of forms $\alpha_{t}=$ $\alpha_{0}+t d f$, we get the required statement.

Lemma 6.1. Suppose that on $W^{\prime}$ we have an open set $U$ with local coordinates $\left(\bar{y}_{1}, \ldots, \bar{y}_{r}\right)$ and an open set $V$ endowed with 1-forms

$$
\gamma_{U}=F_{1} d \bar{y}_{1}+\cdots+F_{\nu} d \bar{y}_{r}, \quad \gamma_{V}=G_{1} d \bar{y}_{0}+\cdots+G_{\nu} d \bar{y}_{r},
$$

that are equal on the intersection $U \cap V$. Then there exist functions $E_{0}, \ldots, E_{r}$ defined on $U \cup V$ satisfying

$$
\gamma_{U}=E_{0} d \bar{y}_{0}+\cdots+\left.E_{r} d \bar{y}_{r}\right|_{U}, \quad \gamma_{V}=E_{0} d \bar{y}_{0}+\cdots+\left.E_{r} d \bar{y}_{r}\right|_{V} .
$$

Proof. The statement is trivial if $U \cap V=\emptyset$. Assume $U \cap V \neq \emptyset$. According to constraints (6.10), the form $\gamma_{U}$ does not change under the addition of terms proportional to $\bar{z}_{0} d \bar{y}_{0}+\cdots+\bar{z}_{r} d \bar{y}_{y}$. We are looking for a function $A: U \rightarrow \mathbb{R}$ that satisfies

$$
A \bar{z}_{0}=G_{0}, \quad F_{1}+A \bar{z}_{1}=G_{1}, \quad \ldots \quad F_{r}+A \bar{z}_{r}=G_{r}
$$

on $U \cap V$. Although it is overdetermined system, due to the condition that $\gamma_{U}=\gamma_{V}$ it has an unique solution. Indeed, on $U$ we have $\bar{z}_{0} \neq 0$ and $\partial \bar{y}_{0} / \partial \bar{y}_{i}=-\bar{z}_{i} / \bar{z}_{0}$ (see Remark 5.2). Therefore, the equality $\gamma_{U}=\gamma_{V}$ implies the following compatibility conditions

$$
F_{1}=-G_{0} \frac{\bar{z}_{1}}{\bar{z}_{0}}+G_{1}, \ldots, F_{r}=-G_{0} \frac{\bar{z}_{r}}{\bar{z}_{0}}+G_{r}, \quad \bar{y} \in U \cap V .
$$


From (6.13), we obtain that $A=G_{0} / \bar{z}_{0}: U \cap V \rightarrow \mathbb{R}$ is a solution of (6.12). Now we take an arbitrary extension of $A$ from $U \cap V$ to $U$ and define

$$
E_{0}\left|U=A, E_{0}\right|_{V}=G_{0}, \quad E_{i}=F_{i}+\left.A \bar{z}_{i}\right|_{U}, E_{i}=\left.G_{i}\right|_{V}, \quad i=1, \ldots, r .
$$

\section{References}

[1] V.I. Arnold, Mathematical methods of classical mechanics, Graduate Texts in Mathematics, 60, Springer-Verlag, New York, 1989.

[2] A. Banyaga and P. Molino, Géométrie des formes de contact complétement intégrables de type torique, Séminare Gaston Darboux, Montpellier (1991-92), 1-25. See also: Complete Integrability in Contact Geometry, Penn State preprint PM 197, 1996.

[3] O.I. Bogoyavlenskij, Extended integrability and bi-Hamiltonian systems, Comm. Math. Phys. 196(1) (1998), 19-51.

[4] A.V. Bolsinov and B. Jovanović, Non-commutative integrability, moment map and geodesic flows, Ann. Global Anal. Geom. 23(4) (2003), 305-322, arXiv: math-ph/0109031.

[5] C.P. Boyer and K. Galicki, A note on toric contact geometry, J. Geom. Phys. 35 (2000), 288-298, arXiv:math/9907043.

[6] W. Boothby and H.C. Wang, On contact manifolds, Ann. Math. 68 (1958), 721-734.

[7] P. Dazord and T. Delzant, Le probleme general des variables actions-angles, J. Diff. Geom. 26(2) (1987), 223-251.

[8] C.M. Davison, H.R. Dullin and A.V. Bolsinov, Geodesics on the ellipsoid and monodromy, J. Geom. Phys. 57(12) (2007), 2437-2454.

[9] V. Dragovic and M. Radnovic, Integrable billiards and quadrics, Uspekhi Mat. Nauk, 65(2) (2010), 133-194 (Russian); English translation in Russian Math. Surveys, 65(2) (2010), 319-379.

[10] V. Dragovic and M. Radnovic, Ellipsoidal billiards in pseudo-Euclidean spaces and relativitic quadrics, Adv. Math. 231(3-4) (2012), 1173-1201, arXiv:1108.4552

[11] J.J. Duistermaat, On global action-angle coordinates, Comm. Pure Appl. Math. 33(6) (1980), 687-706.

[12] Yu. N. Fedorov, Yu. N. and B. Jovanović, Nonholonomic LR systems as generalized Chaplygin systems with an invariant measure and geodesic flows on homogeneous spaces, J. Nonlinear Sci. 14 (2004), 341-381, arXiv:math-ph/0307016.

[13] E. Fiorani and G. Sardanashvily, Global action-angle coordinates for completely integrable systems with noncompact invariant submanifolds, J. Math. Phys. 48(3) (2007), 032901, 9 p, arXiv:math/0610790 [math.DS].

[14] H. Geiges, An introduction to contact topology, Cambridge Studies in Advanced Mathematics, 109, Cambridge University Press, Cambridge, 2008.

[15] J.W. Grey, Some global properties of contact structures, Ann. Math. 69 (1959), 421-450.

[16] B. Jovanović, Symmetries and integrability, Publ. Inst. Math. (Beograd) (N.S.) 84(98) (2008), 1-36, arXiv:0812.4398 [math.SG].

[17] B. Jovanović, Hamiltonization and integrability of the Chaplygin sphere in $R^{n}$, J. Nonlinear Sci. 20 (2010) 569-593, arXiv:0902.4397 [math-ph]. 
[18] B. Khesin and S. Tabachnikov, Pseudo-Riemannian geodesics and billiards, Adv. Math. 221 (2009), 1364-1396, arXiv: math/0608620 [math.DG].

[19] B. Khesin and S. Tabachnikov, Contact complete integrability, regular and chaotic dynamics, 15(4-5) (2010), 504-520, arXiv:0910.0375 [math.SG].

[20] V.V. Kozlov, Symmetries, topology and resonances in Hamiltonian mechanics, Ergebnisse der Mathematik und ihrer Grenzgebiete (3), 31. Springer-Verlag, Berlin, 1996.

[21] C. Laurent-Gengoux, E. Miranda and P. Vanhaecke, Action-angle coordinates for integrable systems on Poisson manifolds, Int. Math. Res. Not., doi:10.1093/imrn/rnq130, arXiv: arxiv.0805.1679 [math.SG].

[22] E. Lerman, Contact toric manifolds, J. Symplectic Geom. 1(4) (2002), 785-828, arXiv:math/0107201 [math.SG].

[23] E. Lerman, Contact fiber bundles, J. Geom. Phys. 49 (2004), 52-66, arXiv:math/0301137 [math.DG].

[24] P. Libermann, Pseudogroupes infinitesimaux attaches aux pseudogroupes de Lie, Bull. Soc. Math. France 87 (1959), 409-425.

[25] P. Libermann and C. Marle, Symplectic geometry, Analytical mechanics, Riedel, Dordrecht, 1987.

[26] P. Libermann, Cartan-Darboux theorems for Pfaffian forms on foliated manifolds, Proc. VIth Int. Colloq. Dif. Geom. Santiago 1989, 125-144. See also: On symplectic and contact groupoids, Differential Geometry and Its Application, Proc. Conf. Opava, 1992, 29-45.

[27] P. Libermann, Legendre foliations on contact manifolds, Diff. Geom. Appl. 1 (1991), $57-76$.

[28] R. Lutz, Sur la geometrie des structures de contact invariantes, Annales de l'institut Fourier 29(1) (1979), 283-306.

[29] E. Miranda, A normal form theorem for integrable systems on contact manifolds, Proceeding of XIII Fall Workshop on Geometry and Physics, Murcia, September 2022, 2004, Publ. de la RSME, 9 (2005), 240-246.

[30] A.S. Mishchenko and A.T. Fomenko, Generalized Liouville method of integration of Hamiltonian systems, Funktsional Anal. i. Prilozhen. 12(2) (1978), 46-56 (Russian); English translation: Funct. Anal. Appl. 12 (1978), 113-121.

[31] N.N. Nehoroshev, Action-angle variables and their generalization. Tr. Mosk. Mat. O.va. 26 (1972), 181-198 (Russian); English translation: Trans. Moscow Math. Soc. 26 (1972), 180-198.

[32] M.Y. Pang, The structure of Legendre foliations, Trans. Amer. Math. Soc. 320 (1990), $417-455$.

[33] C.B. Thomas, Almost regular contact manifolds, J. Diff. Geom. 11 (1976), 521-533.

[34] A.P. Veselov, Integrable mappings, Uspekhi Mat. Nauk 46(5) (1991), 3-45 (Russian); English translation in Russian Math. Surveys 46(5) (1991), 1-51.

[35] T. Yamazaki, A construction of K-contact manifolds by a fiber join, Tohoku Math. J. 51 (1999), 433-446.

[36] N.T. Zung, Torus actions and integrable systems, in 'Topological Methods in the Theory of Integrable Systems', A.V. Bolsinov, A.T. Fomenko, A. A. Oshemkov (eds.), 289-328, Cambridge Scientific Publ., (2006), arXiv: math.DS/0407455 
Mathematical Institute, Serbian Academy of Sciences and Arts, Kneza Mihaila 36, 11000 Belgrade, Serbia

bozaj@mi.sanu.ac.rs

Received 01/24/2011, accepted 04/21/2011

This research was supported by the Serbian Ministry of Science Project 174020, Geometry and Topology of Manifolds, Classical Mechanics and Integrable Dynamical Systems. The part of the paper is written during author's visiting SISSA in July 2010. Author would like to thank Professor Dubrovin for kind hospitality. 
